\title{
Structure of HDL: Particle Subclasses and Molecular Components
}

\author{
Anatol Kontush, Mats Lindahl, Marie Lhomme, Laura Calabresi, \\ M. John Chapman, and W. Sean Davidson
}

\section{Contents}

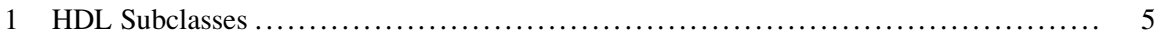

2 Molecular Components of HDL .......................................... 7

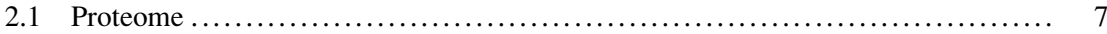

2.1.1 Major Protein Components ..................................... 7

2.1.2 Protein Isoforms, Translational and Posttranslational Modifications ....... 19

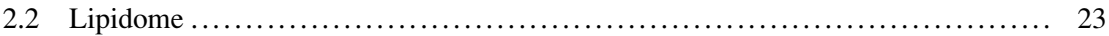

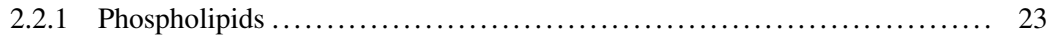

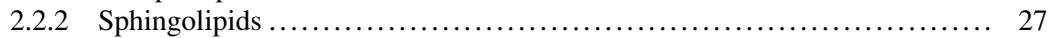

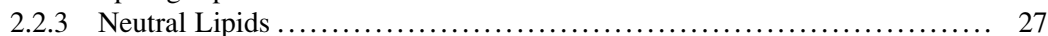

A. Kontush $(\varangle) \cdot$ M. Lhomme • M.J. Chapman

National Institute for Health and Medical Research (INSERM), UMR-ICAN 1166, Paris, France

University of Pierre and Marie Curie - Paris 6, Paris, France

Pitié - Salpétrière University Hospital, Paris, France

ICAN, Paris, France

e-mail: anatol.kontush@upmc.fr; m.lhomme@ican-institute.org; john.chapman@upmc.fr

\section{Lindahl}

Department of Clinical and Experimental Medicine, Linköping University, Linköping, Sweden e-mail: mats.lindahl@liu.se

\section{Calabresi}

Department of Pharmacological and Biomolecular Sciences, Center E. Grossi Paoletti,

University of Milan, Milan, Italy

e-mail: laura.calabresi@unimi.it

W.S. Davidson

Department of Pathology and Laboratory Medicine, University of Cincinnati, Cincinnati, OH 45237, USA

e-mail: davidswm@ucmail.uc.edu 


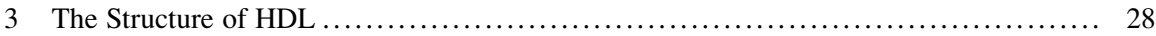

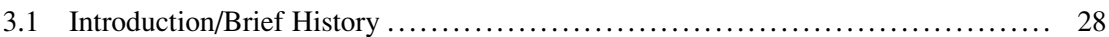

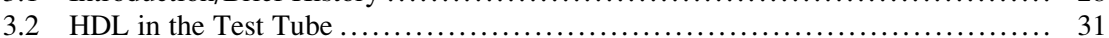

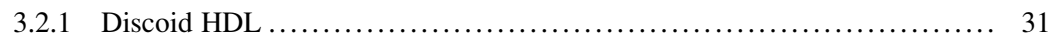

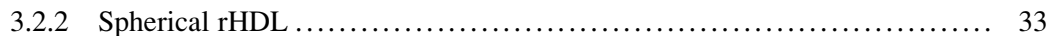

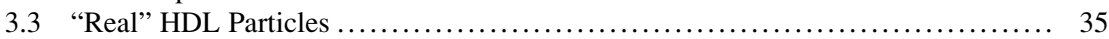

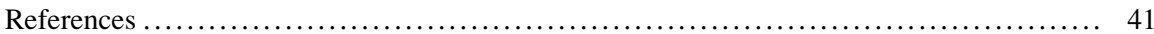

\section{Abstract}

A molecular understanding of high-density lipoprotein (HDL) will allow a more complete grasp of its interactions with key plasma remodelling factors and with cell-surface proteins that mediate HDL assembly and clearance. However, these particles are notoriously heterogeneous in terms of almost every physical, chemical and biological property. Furthermore, HDL particles have not lent themselves to high-resolution structural study through mainstream techniques like nuclear magnetic resonance and X-ray crystallography; investigators have therefore had to use a series of lower resolution methods to derive a general structural understanding of these enigmatic particles. This chapter reviews current knowledge of the composition, structure and heterogeneity of human plasma HDL. The multifaceted composition of the HDL proteome, the multiple major protein isoforms involving translational and posttranslational modifications, the rapidly expanding knowledge of the HDL lipidome, the highly complex world of HDL subclasses and putative models of HDL particle structure are extensively discussed. A brief history of structural studies of both plasma-derived and recombinant forms of HDL is presented with a focus on detailed structural models that have been derived from a range of techniques spanning mass spectrometry to molecular dynamics.

\section{Keywords}

HDL • Composition • Structure • Heterogeneity • Proteomics • Lipidomics • Proteome $\bullet$ Lipidome $\bullet$ Post-translational $\bullet$ Modifications

High-density lipoprotein (HDL) is a small, dense, protein-rich lipoprotein as compared to other lipoprotein classes, with a mean size of 8-10 $\mathrm{nm}$ and density of $1.063-1.21 \mathrm{~g} / \mathrm{ml}$ (Kontush and Chapman 2012). HDL particles are plurimolecular, quasi-spherical or discoid, pseudomicellar complexes composed predominantly of polar lipids solubilised by apolipoproteins. HDL also contains numerous other proteins, including enzymes and acute-phase proteins, and may contain small amounts of nonpolar lipids. Furthermore, HDL proteins often exist in multiple isoforms and readily undergo posttranslational modification. As a consequence of such diverse compositional features, HDL particles are highly heterogeneous in their structural, chemical and biological properties. This chapter reviews current knowledge of the composition, structure and heterogeneity of human plasma HDL. 


\section{$1 \quad$ HDL Subclasses}

Human plasma HDLs are a highly heterogeneous lipoprotein family consisting of several subclasses differing in density, size, shape and lipid and protein composition (Table 1).

Differences in HDL subclass distribution were first described by Gofman and colleagues in the early 1950s by using analytic ultracentrifugation (De Lalla and Gofman 1954), the gold standard technique for HDL separation. Two HDL subclasses were identified: the less dense $(1.063-1.125 \mathrm{~g} / \mathrm{mL})$, relatively lipidrich form was classified as HDL2 and the more dense $(1.125-1.21 \mathrm{~g} / \mathrm{mL})$, relatively protein-rich form as HDL3. The two major HDL subclasses can be separated by other ultracentrifugation methods, such as rate-zonal ultracentrifugation (Franceschini et al. 1985) or single vertical spin ultracentrifugation (Kulkarni et al. 1997). Ultracentrifugation methods are accurate and precise but require expensive instruments, time and technical skills. A precipitation method has been proposed for HDL2 and HDL3 separation and quantitation (Gidez et al. 1982), which is inexpensive and easier, but with a high degree of interlaboratory variability. HDL2 and HDL3 can be further fractionated in distinct subclasses with different electrophoretic mobilities by non-denaturing polyacrylamide gradient gel electrophoresis (GGE) (Nichols et al. 1986), which separates HDL subclasses on the basis of particle size. Two HDL2 and three HDL3 subclasses have been identified and their particle size characterised by this method: HDL3c, 7.2-7.8 nm diameter; HDL3b, 7.8-8.2 nm; HDL3a, 8.2-8.8 nm; HDL2a, 8.8.$9.7 \mathrm{~nm}$; and HDL2b, 9.7-12.0 nm. The equivalent subclasses of HDL with similar size distribution may be preparatively isolated by isopycnic density gradient ultracentrifugation (Chapman et al. 1981; Kontush et al. 2003).

Agarose gel electrophoresis allows analytical separation of HDL according to surface charge and shape into $\boldsymbol{\alpha}$-migrating particles, which represent the majority of circulating HDL, and pre $\beta$-migrating particles, consisting of nascent discoidal and poorly lipidated HDL. Agarose gels can be stained with Coomassie blue or with anti-apolipoprotein A-I (apoA-I) antibodies, and the relative protein content of the two HDL subclasses can be determined (Favari et al. 2004). The plasma pre $\beta$-HDL concentration can be also quantified using a sandwich enzyme immunoassay (Miida et al. 2003). The assay utilises a monoclonal antibody which specifically recognises apoA-I bound to pre $\beta$-HDL. The agarose gel and the GGE can be combined into a 2-dimensional (2D) electrophoretic method, which separates HDL according to charge in the first run and according to size in the second run. Gels can be stained with apolipoprotein-specific antibodies, typically with anti-apoA-I antibodies, allowing the detection of distinct HDL subclasses (Asztalos et al. 2007). This is by far the method with the highest resolving power: up to 12 distinct apoA-Icontaining HDL subclasses can be identified, referred to as pre $\beta$ (pre $\beta_{1}$ and $\left.\operatorname{pre} \beta_{2}\right), \alpha$ $\left(\alpha_{1}, \alpha_{2}, \alpha_{3}\right.$ and $\left.\alpha_{4}\right)$ and $\operatorname{pre} \alpha\left(\operatorname{pre} \alpha_{1}, \operatorname{pre} \alpha_{2}, \operatorname{pre} \alpha_{3}\right)$ according to their mobility and size (Asztalos and Schaefer 2003a, b).

According to the protein component, HDL can be separated into particles containing apoA-I with (LpA-I:A-II) or without apoA-II (LpA-I) by an 
Table 1 Major HDL subclasses according to different isolation/ separation techniques

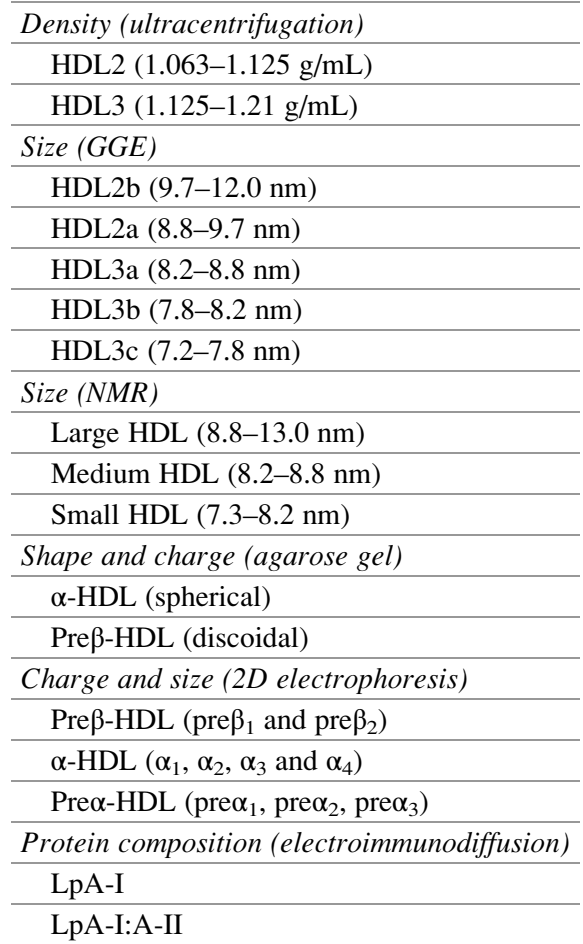

electroimmunodiffusion technique in agarose gels; plasma concentration of LpA-I and LpA-I:A-II can be determined from a calibration curve (Franceschini et al. 2007).

More recently, a nuclear magnetic resonance (NMR) method for HDL subclass analysis has been proposed, based on the concept that each lipoprotein particle of a given size has its own characteristic lipid methyl group NMR signal (Otvos et al. 1992). According to the NMR signals, three HDL subclasses can be identified: large HDL (8.8-13.0 $\mathrm{nm}$ diameter), medium HDL $(8.2-8.8 \mathrm{~nm})$ and small HDL (7.3-8.2 $\mathrm{nm})$; results are expressed as plasma particle concentration. The relative plasma content of small, medium and large HDL (according to the same cut-off) can also be determined by GGE, dividing the absorbance profile into the three size intervals (Franceschini et al. 2007).

HDL subfractionation on an analytical scale has been generally accomplished by the different techniques in academic laboratories; however, clinical interest in HDL heterogeneity has been growing in the last 10 years and a number of laboratory tests for determining HDL subclass distribution are now available, including GGE, NMR and 2D gel electrophoresis (Mora 2009). Whether evaluation of HDL subfractions is performed by academic or commercial laboratories, there are a number of factors that confound the interpretation of the results of such analyses. The number and nomenclature of HDL subclasses are not uniform among the different techniques; 
moreover, each subclass contains distinct subpopulations, as identified by, e.g. 2D electrophoresis. In addition, whereas some methodologies measure HDL subclass concentrations, others describe the percent distribution of the HDL subclasses relative to the total or characterise the HDL distribution by average particle diameter. As a consequence, there is little relation among HDL subfractionation data produced by different analytical techniques. A panel of experts has recently proposed a classification of HDL by physical properties, which integrates terminology from several methods and defines five HDL subclasses, termed very large, large, medium, small and very small HDL (Rosenson et al. 2011). The proposed nomenclature, possibly together with widely accepted standards and quality controls, should help in defining the relationship between HDL subclasses and cardiovascular risk as well as in assessing the clinical effects of HDL modifying drugs.

\section{Molecular Components of HDL}

\subsection{Proteome}

\subsubsection{Major Protein Components}

Proteins form the major structural and functional component of HDL particles. HDL carries a large number of different proteins as compared to other lipoprotein classes (Table 2). HDL proteins can be divided into several major subgroups which include apolipoproteins, enzymes, lipid transfer proteins, acute-phase response proteins, complement components, proteinase inhibitors and other protein components. Whereas apolipoproteins and enzymes are widely recognised as key functional HDL components, the role of minor proteins, primarily those involved in complement regulation, protection from infections and the acute-phase response, has received increasing attention only in recent years, mainly as a result of advances in proteomic technologies (Heinecke 2009; Hoofnagle and Heinecke 2009; Davidsson et al. 2010; Shah et al. 2013). These studies have allowed reproducible identification of more than 80 proteins in human HDL (Heinecke 2009; Hoofnagle and Heinecke 2009; Davidsson et al. 2010; Shah et al. 2013) (for more details see the HDL Proteome Watch at http://homepages.uc.edu/ davidswm/HDLproteome. html). Numerous proteins involved in the acute-phase response, complement regulation, proteinase inhibition, immune response and haemostasis were unexpectedly found as components of normal human plasma HDL, raising the possibility that HDL may play previously unsuspected roles in host defence mechanisms and inflammation (Hoofnagle and Heinecke 2009).

Importantly, the composition of the HDL proteome may depend on the method of HDL isolation. Indeed, ultracentrifugation in highly concentrated salt solutions of high ionic strength can remove some proteins from HDL, whereas other methods of HDL isolation (gel filtration, immunoaffinity chromatography, precipitation) provide HDL extensively contaminated with plasma proteins or subject HDL to unphysiological conditions capable of modifying its structure and/or composition 
Table 2 Major components of the HDL proteome

\begin{tabular}{|c|c|c|c|}
\hline Protein & $\mathrm{M}_{\mathrm{r}}, \mathrm{kDa}$ & Major function & $\begin{array}{l}\text { Number of proteomic } \\
\text { studies in which the } \\
\text { protein was detected }^{\text {a }}\end{array}$ \\
\hline \multicolumn{4}{|l|}{ Apolipoproteins } \\
\hline ApoA-I & 28 & $\begin{array}{l}\text { Major structural and functional } \\
\text { apolipoprotein, LCAT activator }\end{array}$ & 14 \\
\hline ApoA-II & 17 & $\begin{array}{l}\text { Structural and functional } \\
\text { apolipoprotein }\end{array}$ & 13 \\
\hline ApoA-IV & 46 & $\begin{array}{l}\text { Structural and functional } \\
\text { apolipoprotein }\end{array}$ & 14 \\
\hline ApoC-I & 6.6 & $\begin{array}{l}\text { Modulator of CETP activity, } \\
\text { LCAT activator }\end{array}$ & 12 \\
\hline ApoC-II & 8.8 & Activator of LPL & 12 \\
\hline ApoC-III & 8.8 & Inhibitor of LPL & 14 \\
\hline ApoC-IV & 11 & Regulates TG metabolism & 6 \\
\hline ApoD & 19 & $\begin{array}{l}\text { Binding of small hydrophobic } \\
\text { molecules }\end{array}$ & 11 \\
\hline ApoE & 34 & $\begin{array}{l}\text { Structural and functional } \\
\text { apolipoprotein, ligand for LDL-R } \\
\text { and LRP }\end{array}$ & 13 \\
\hline ApoF & 29 & Inhibitor of CETP & 8 \\
\hline ApoH & 38 & $\begin{array}{l}\text { Binding of negatively charged } \\
\text { molecules }\end{array}$ & 8 \\
\hline ApoJ & 70 & $\begin{array}{l}\text { Binding of hydrophobic molecules, } \\
\text { interaction with cell receptors }\end{array}$ & 11 \\
\hline ApoL-I & $44 / 46$ & Trypanolytic factor of human serum & 14 \\
\hline ApoM & 25 & $\begin{array}{l}\text { Binding of small hydrophobic } \\
\text { molecules }\end{array}$ & 12 \\
\hline \multicolumn{4}{|l|}{ Enzymes } \\
\hline LCAT & 63 & $\begin{array}{l}\text { Esterification of cholesterol to } \\
\text { cholesteryl esters }\end{array}$ & 4 \\
\hline PON1 & 43 & Calcium-dependent lactonase & 12 \\
\hline $\begin{aligned} & \text { PAF-AH } \\
&\left(\mathrm{LpPLA}_{2}\right)\end{aligned}$ & 53 & $\begin{array}{l}\text { Hydrolysis of short-chain oxidised } \\
\text { phospholipids }\end{array}$ & \\
\hline GSPx-3 & 22 & $\begin{array}{l}\text { Reduction of hydroperoxides by } \\
\text { glutathione }\end{array}$ & \\
\hline \multicolumn{4}{|c|}{ Lipid transfer proteins } \\
\hline PLTP & 78 & $\begin{array}{l}\text { Conversion of HDL into larger and } \\
\text { smaller particles, transport of LPS }\end{array}$ & 5 \\
\hline CETP & 74 & $\begin{array}{l}\text { Heteroexchange of CE and TG and } \\
\text { homoexchange of PL between HDL } \\
\text { and apoB-containing lipoproteins }\end{array}$ & 3 \\
\hline \multicolumn{4}{|c|}{ Acute-phase proteins } \\
\hline SAA1 & 12 & Major acute-phase reactant & 10 \\
\hline SAA4 & 15 & Minor acute-phase reactant & 10 \\
\hline $\begin{array}{l}\text { Alpha-2-HS- } \\
\text { glycoprotein }\end{array}$ & 39 & Negative acute-phase reactant & 9 \\
\hline
\end{tabular}


Table 2 (continued)

\begin{tabular}{|c|c|c|c|}
\hline Protein & $\mathrm{M}_{\mathrm{r}}, \mathrm{kDa}$ & Major function & $\begin{array}{l}\text { Number of proteomic } \\
\text { studies in which the } \\
\text { protein was detected }\end{array}$ \\
\hline $\begin{array}{l}\text { Fibrinogen } \\
\text { alpha chain }\end{array}$ & 95 & $\begin{array}{l}\text { Precursor of fibrin, cofactor in } \\
\text { platelet aggregation }\end{array}$ & 10 \\
\hline \multicolumn{4}{|c|}{ Complement components } \\
\hline $\mathrm{C} 3$ & 187 & Complement activation & 9 \\
\hline \multicolumn{4}{|c|}{ Proteinase inhibitors } \\
\hline $\begin{array}{l}\text { Alpha-1- } \\
\text { antitrypsin }\end{array}$ & 52 & Inhibitor of serine proteinases & 11 \\
\hline Hrp & 39 & $\begin{array}{l}\text { Decoy substrate to prevent } \\
\text { proteolysis }\end{array}$ & 10 \\
\hline \multicolumn{4}{|l|}{ Other proteins } \\
\hline Transthyretin & 55 & $\begin{array}{l}\text { Thyroid hormone binding and } \\
\text { transport }\end{array}$ & 12 \\
\hline Serotransferrin & 75 & Iron binding and transport & 10 \\
\hline $\begin{array}{l}\quad \text { Vitamin } \\
\text { D-binding } \\
\text { protein }\end{array}$ & 58 & Vitamin D binding and transport & 10 \\
\hline $\begin{array}{l}\text { Alpha-1B- } \\
\text { glycoprotein }\end{array}$ & 54 & Unknown & 9 \\
\hline Hemopexin & 52 & Heme binding and transport & 8 \\
\hline
\end{tabular}

${ }^{\mathrm{a}}$ Out of total of 14 proteomic studies according to Shah et al. (2013). Only proteins detected in more than $50 \%$ of the studies are listed, together with seven others previously known to be associated with HDL (apoC-IV, apoH, LCAT, PAF-AH, GSPx-3, PLTP, CETP)

$C E$ cholesteryl ester, $C E T P$ cholesteryl ester transfer protein, GSPx-3 glutathione selenoperoxidase 3, $H r p$ haptoglobin-related protein, $L D L-R$ LDL receptor, $L C A T$ lecithin/cholesterol acyltransferase, $L P L$ lipoprotein lipase, $L P P L A 2$ lipoprotein-associated phospholipase A2, $L R P$ LDL receptor-related protein, $P A F-A H$ platelet-activating factor acetyl hydrolase, $P L$ phospholipid, PLTP phospholipid transfer protein, $P O N 1$ paraoxonase $1, S A A$ serum amuloid A, $T G$ triglyceride

(e.g. extreme $\mathrm{pH}$ and ionic strength involved in immunoaffinity separation). Thus, proteomics of apoA-I-containing fractions isolated from human plasma by a non-denaturing approach of fast protein liquid chromatography (FPLC) reveal the presence of up to 115 individual proteins per fraction, only up to 32 of which were identified as HDL-associated proteins in ultracentrifugally isolated HDL (Collins et al. 2010). Indeed, co-elution with HDL of plasma proteins of matching size is inevitable in FPLC-based separation; the presence of a particular protein across a range of HDL-containing fractions of different size isolated by FPLC on the basis of their association with phospholipid would however suggest that such a protein is indeed associated with HDL (Gordon et al. 2010). Remarkably, several of the most abundant plasma proteins, including albumin, haptoglobin and alpha-2-macroglobulin, are indeed present in all apoA-I-containing fractions isolated by FPLC (Collins et al. 2010), suggesting their partial association with HDL by a non-specific, low-affinity binding. 


\section{Apolipoproteins}

Apolipoprotein A-I is the major structural and functional HDL protein which accounts for approximately $70 \%$ of total HDL protein. Almost all HDL particles are believed to contain apoA-I (Asztalos and Schaefer 2003a, b; Schaefer et al. 2010). Major functions of apoA-I involve interaction with cellular receptors, activation of lecithin/cholesterol acyltransferase (LCAT) and endowing HDL with multiple anti-atherogenic activities. Circulating apoA-I represents a typical amphipathic protein that lacks glycosylation or disulfide linkages and contains eight alpha-helical amphipathic domains of 22 amino acids and two repeats of 11 amino acids. As a consequence, apoA-I binds avidly to lipids and possesses potent detergent-like properties. ApoA-I readily moves between lipoprotein particles and is also found in chylomicrons and very low-density lipoprotein (VLDL). As for many plasma apolipoproteins, the main sites for apoA-I synthesis and secretion are the liver and small intestine.

ApoA-II is the second major HDL apolipoprotein which represents approximately $15-20 \%$ of total HDL protein. About a half of HDL particles may contain apoA-II (Duriez and Fruchart 1999). ApoA-II is more hydrophobic than apoA-I and circulates as a homodimer composed of two identical polypeptide chains (Shimano 2009; Puppione et al. 2010) connected by a disulfide bridge at position 6 (Brewer et al. 1972). ApoA-II equally forms heterodimers with other cysteine-containing apolipoproteins (Hennessy et al. 1997) and is predominantly synthesised in the liver but also in the intestine (Gordon et al. 1983).

ApoA-IV, an O-linked glycoprotein, is the most hydrophilic apolipoprotein which readily exchanges between lipoproteins and also circulates in a free form. ApoA-IV contains thirteen 22-amino acid tandem repeats, nine of which are highly alpha-helical; many of these helices are amphipathic and may serve as lipid-binding domains. In man, apoA-IV is synthesised in the intestine and is secreted into the circulation with chylomicrons.

ApoCs form a family of small exchangeable apolipoproteins primarily synthesised in the liver. ApoC-I is the smallest apolipoprotein which associates with both HDL and VLDL and can readily exchange between them. ApoC-I carries a strong positive charge and can thereby bind free fatty acids and modulate activities of several proteins involved in HDL metabolism. Thus, apoC-I is involved in the activation of LCAT and inhibition of hepatic lipase and cholesteryl ester transfer protein (CETP). ApoC-II functions as an activator of several triacylglycerol lipases and is associated with HDL and VLDL. ApoC-III is predominantly present in VLDL with small amounts found in HDL. The protein inhibits lipoprotein lipase (LPL) and hepatic lipase and decreases the uptake of chylomicrons by hepatic cells. ApoC-IV induces hypertriglyceridemia when overexpressed in mice (Allan and Taylor 1996; Kim et al. 2008). In normolipidemic plasma, greater than $80 \%$ of the protein resides in VLDL, with most of the remainder in HDL. The HDL content of apoC-IV is much lower as compared to the other apoC proteins.

ApoD is a glycoprotein mainly associated with HDL (McConathy and Alaupovic 1973). The protein is expressed in many tissues, including liver and intestine. ApoD does not possess a typical apolipoprotein structure and belongs to 
the lipocalin family which also includes retinol-binding protein, lactoglobulin and uteroglobulin. Lipocalins are small lipid transfer proteins with a limited amino acid sequence identity but with a common tertiary structure. Lipocalins share a structurally conserved beta-barrel fold, which in many lipocalins bind hydrophobic ligands. As a result, apoD transports small hydrophobic ligands, with a high affinity for arachidonic acid (Rassart et al. 2000). In plasma, apoD forms disulfide-linked homodimers and heterodimers with apoA-II.

ApoE is a key structural and functional glycoprotein component of HDL despite its much lower content in HDL particles as compared to apoA-I (Utermann 1975). The major fraction of circulating apoE is carried by triglyceride-containing lipoproteins where it serves as a ligand for apoB/apoE receptors and ensures lipoprotein binding to cell-surface glycosaminoglycans. Similar to apoA-I and apoA-II, apoE contains eight amphipathic alpha-helical repeats and displays detergent-like properties towards phospholipids (Lund-Katz and Phillips 2010). ApoE is synthesised in multiple tissues and cell types, including liver, endocrine tissues, central nervous system and macrophages.

ApoF is a sialoglycoprotein present in human HDL and low-density lipoprotein (LDL) (Olofsson et al. 1978), also known as lipid transfer inhibitor protein (LTIP) as a consequence of its ability to inhibit CETP. ApoF is synthesised in the liver and heavily glycosylated with both O- and N-linked sugar groups. Such glycosylation renders the protein highly acidic and results in a molecular mass some $40 \%$ greater than predicted (Lagor et al. 2009).

ApoH, also known as beta-2-glycoprotein 1, is a multifunctional $\mathrm{N}$ - and $\mathrm{O}$-glycosylated protein. ApoH binds to various kinds of negatively charged molecules, primarily to cardiolipin, and may prevent activation of the intrinsic blood coagulation cascade by binding to phospholipids on the surface of damaged cells. Such binding properties are ensured by a positively charged domain. $\mathrm{ApoH}$ regulates platelet aggregation and is expressed by the liver.

ApoJ (also called clusterin and complement-associated protein SP-40,40) is an antiparallel disulfide-linked heterodimeric glycoprotein. Human apoJ consists of two subunits designated alpha (34-36 kDa) and beta (36-39 kDa) which share limited homology (de Silva et al. 1990a, b) and are linked by five disulfide bonds. The distinct structure of apoJ allows binding of both a wide spectrum of hydrophobic molecules on the one hand and of specific cell-surface receptors on the other.

ApoL-I is a key component of the trypanolytic factor of human serum associated with HDL (Duchateau et al. 1997). ApoL-I possesses a glycosylation site and shares structural and functional similarities with intracellular apoptosis-regulating proteins of the Bcl-2 family. ApoL-I displays high affinity for phosphatidic acid and cardiolipin (Zhaorigetu et al. 2008) and is expressed in pancreas, lung, prostate, liver, placenta and spleen.

ApoM is an apolipoprotein found mainly in HDL (Axler et al. 2007) which possesses an eight stranded antiparallel beta-barrel lipocalin fold and a hydrophobic pocket that ensures binding of small hydrophobic molecules, primarily sphingosine-1-phosphate (S1P) (Ahnstrom et al. 2007; Christoffersen et al. 2011). ApoM reveals $19 \%$ homology with apoD, another apolipoprotein member of the lipocalin 
family (Sevvana et al. 2009), and is synthesised in the liver and kidney. The binding of apoM to lipoproteins is assured by its hydrophobic N-terminal signal peptide which is retained on secreted apoM, a phenomenon atypical for plasma apolipoproteins (Axler et al. 2008; Christoffersen et al. 2008; Dahlback and Nielsen 2009).

ApoO, a minor HDL component expressed in several human tissues (Lamant et al. 2006), is present in HDL, LDL and VLDL, belongs to the proteoglycan family and contains chondroitin sulphate chains, a unique feature distinguishing it from other apolipoproteins. The physiological function of apoO remains unknown (Nijstad et al. 2011).

Minor apolipoprotein components isolated within the density range of HDL are also exemplified by apoB and apo(a), which reflect the presence of lipoprotein (a) and result from overlap in the hydrated densities of large, light HDL2 and lipoprotein (a) (Davidson et al. 2009).

\section{Enzymes}

LCAT catalyses the esterification of cholesterol to cholesteryl esters in plasma lipoproteins, primarily in HDL but also in apoB-containing particles. Approximately $75 \%$ of plasma LCAT activity is associated with HDL. In plasma, LCAT is closely associated with apoD, which frequently co-purify (Holmquist 2002). The LCAT gene is primarily expressed in the liver and, to a lesser extent, in the brain and testes. The LCAT protein is heavily N-glycosylated. The tertiary structure of LCAT is maintained by two disulfide bridges and involves an active site covered by a lid (Rousset et al. 2009). LCAT contains two free cysteine residues at positions 31 and 184.

Human paraoxonases (PON) are calcium-dependent lactonases PON1, PON2 and PON3 (Goswami et al. 2009). In the circulation, PON1 is almost exclusively associated with HDL; such association is mediated by HDL surface phospholipids and requires the hydrophobic leader sequence retained in the secreted PON1. Human PON1 is largely synthesised in the liver but also in the kidney and colon (Mackness et al. 2010). Hydrolysis of homocysteine thiolactone has been proposed to represent a major physiologic function of PON1 (Jakubowski 2000). The name "PON" however reflects the ability of PON1 to hydrolyse the organophosphate substrate paraoxone together with other organophosphate substrates and aromatic carboxylic acid esters. Catalytic activities of the enzyme involve reversible binding to the substrate as the first step of hydrolytic cleavage. PON1 is structurally organised as a six-bladed beta-propeller, with each blade consisting of four betasheets (Harel et al. 2004). Two calcium atoms needed for the stabilisation of the structure and the catalytic activity of the enzyme are located in the central tunnel of the enzyme. Three helices, located at the top of the propeller, are involved in the anchoring of PON1 to HDL. The enzyme is N-glycosylated and may contain a disulfide bond. PON2, another member of the PON family, is an intracellular enzyme not detectable in serum despite its expression in many tissues, including the brain, liver, kidney and testis. The enzyme hydrolyses organophosphate substrates and aromatic carboxylic acid esters. PON3 possesses properties which 
are similar to those of PON1, such as requirement for calcium, N-glycosylation, secretion in the circulation with retained signal peptide and association with HDL. PON3 displays potent lactonase, limited arylesterase and no PON activities and is predominantly expressed in the liver.

Platelet-activating factor acetyl hydrolase (PAF-AH) equally termed lipoprotein-associated phospholipase $\mathrm{A}_{2}$ (LPPLA2) is a calcium-independent, N-glycosylated enzyme, which degrades PAF by hydrolysing the sn-2 ester bond to yield biologically inactive lyso-PAF (Mallat et al. 2010). The enzyme cleaves phospholipid substrates with a short residue at the sn-2 position and can therefore hydrolyse proinflammatory oxidised short-chain phospholipids; however, it is inactive against long-chain non-oxidised phospholipids. PAF-AH is synthesised throughout the brain, white adipose tissue and placenta. Macrophages represent the most important source of the circulating enzyme (McIntyre et al. 2009). Plasma PAF-AH circulates in association with LDL and HDL particles, with the majority of the enzyme bound to small, dense LDL and to lipoprotein (a) (Tselepis et al. 1995). The crystal structure of PAF-AH reveals a typical lipase alpha/beta-hydrolase fold and a catalytic triad (Samanta and Bahnson 2008). The active site is close to the lipoprotein surface and at the same time accessible to the aqueous phase. Two clusters of hydrophobic residues build a lipid-binding domain ensuring association with lipoproteins.

Plasma glutathione selenoperoxidase 3 (GSPx-3), also called glutathione peroxidase 3, is distinct from two other members of the GSPx family termed GSPX-1 and GSPx-2 which represent erythrocyte and liver cytosolic enzymes. All GSPx enzymes protect biomolecules from oxidative damage by catalysing the reduction of hydrogen peroxide, lipid peroxides and organic hydroperoxide, in a reaction involving glutathione. Human GSPx-3 is a homotetrameric protein containing selenium as a selenocysteine residue at position 73. Human GSPX-3 is synthesised in the liver, kidney, heart, lung, breast and placenta. In plasma, GSPX-3 is exclusively associated with HDL (Chen et al. 2000).

\section{Lipid Transfer Proteins}

Phospholipid transfer protein (PLTP) belongs to the bactericidal permeabilityincreasing protein (BPI)/lipopolysaccharide (LPS)-binding protein (LBP)/Plunc superfamily of proteins. PLTP is synthesised in the placenta, pancreas, lung, kidney, heart, liver, skeletal muscle and brain. In the circulation, PLTP is primarily associated with HDL and converts it into larger and smaller particles. PLTP also plays a role in extracellular phospholipid transport and can bind LPS. The protein contains multiple glycosylation sites and is stabilised by a disulfide bond. PLTP is a positive acute-phase reactant with a potential role in the innate immune system.

CETP equally belongs to the BPI/LBP/Plunc superfamily and contains multiple $\mathrm{N}$-glycosylation sites. It is primarily expressed by the liver and adipose tissue. In the circulation, CETP shuttles between HDL and apoB-containing lipoproteins and facilitates the bidirectional transfer of cholesteryl esters and triglycerides between them. The structure of CETP includes a hydrophobic tunnel filled with two cholesteryl ester molecules and plugged by an amphiphilic phosphatidylcholine 
(PC) molecule at each end (Qiu et al. 2007). Such interactions additionally endow CETP with PC transfer activity. CETP may also undergo conformational changes to accommodate lipoprotein particles of different sizes (Qiu et al. 2007).

\section{Acute-Phase Response Proteins}

Positive acute-phase response proteins, whose plasma concentrations are markedly elevated by acute inflammation, form a large family of HDL-associated proteins (Vaisar et al. 2007; Heinecke 2009). Under normal conditions, the content of such proteins in HDL is however much lower as compared to apolipoproteins. On the other hand, plasma levels of several HDL apolipoproteins, such as apoA-I and apoA-IV, are reduced during the acute-phase response (Navab et al. 2004); such proteins can therefore be considered as negative acute-phase response proteins.

Serum amyloid A (SAA) proteins, major acute-phase reactants, are secreted during the acute phase of the inflammatory response. In humans, three SAA isoforms, SAA1, SAA2 and SAA4, are produced predominantly by the liver. Hepatic expression of SAA1 and SAA2 in the liver is induced during the acutephase reaction, resulting in increase in their circulating levels by as much as 1,000fold from basal concentrations of about $1-5 \mathrm{mg} / \mathrm{l}$ (Khovidhunkit et al. 2004). By contrast, SAA4 is expressed constitutively in the liver and is therefore termed constitutive SAA. SAA1, the major member of this family, is predominantly carried by HDL in human, rabbit and murine plasma (Hoffman and Benditt 1982; Marhaug et al. 1982; Cabana et al. 1996). In the circulation, SAA1 does not exist in a free form and associates with non-HDL lipoproteins in the absence of HDL (Cabana et al. 2004).

LBP is an acute-phase glycoprotein capable of binding the lipid A moiety of LPS of Gram-negative bacteria and facilitating LPS diffusion (Wurfel et al. 1994). LBP/LPS complexes appear to interact with the CD14 receptor to enhance cellular responses to LPS. LBP also binds phospholipids, thereby acting as a lipid exchange protein (Yu et al. 1997), and belongs to the same BPI/LBP/Plunc protein superfamily as PLTP and CETP.

Fibrinogen is a common acute-phase protein and a cofactor in platelet aggregation synthesised by the liver, which is converted by thrombin into fibrin during blood coagulation. Fibrinogen is a disulfide-linked heterohexamer which contains two sets of three non-identical chains (alpha, beta and gamma).

Alpha-1-acid glycoprotein 2, equally termed orosomucoid-2, belongs to the calycin protein superfamily which also includes lipocalins and fatty acid-binding proteins. The protein appears to modulate activity of the immune system during the acute-phase reaction. In plasma, the protein is $\mathrm{N}$-glycosylated and stabilised by disulfide bonds.

Alpha-2-HS-glycoprotein (fetuin-A) promotes endocytosis, possesses opsonic properties and influences the mineral phase of bone. The protein shows affinity for calcium and barium ions and contains two chains, $\mathrm{A}$ and $\mathrm{B}$, which are held together by a single disulfide bond. Alpha-2-HS-glycoprotein is synthesised in the liver and secreted into plasma. 


\section{Complement Components}

Several complement components associate with HDL. Complement component 3 (C3) plays a central role in the activation of the complement system through both classical and alternative activation pathways. C3 exists in a form of two chains, beta and alpha, linked by a disulfide bond. C4 is a key component involved in the activation of the classical pathway of the complement system, which circulates as a disulfide-linked trimer of alpha, beta and gamma chains. C4b-binding protein controls the classical pathway of complement activation and binds as a cofactor to $\mathrm{C} 3 \mathrm{~b} / \mathrm{C} 4 \mathrm{~b}$ inactivator, which then hydrolyses complement fragment C4b. C9 is a pore-forming subunit of the membrane attack complex that provides an essential contribution to the innate and adaptive immune response by forming pores in the plasma membrane of target cells.

Vitronectin is another HDL-associated protein involved in complement regulation. The protein belongs to cell-to-substrate adhesion molecules present in serum and tissues, which interact with glycosaminoglycans and proteoglycans and can be recognised by members of the integrin family. In complement regulation, vitronectin serves as an inhibitor of the membrane-damaging effect of the terminal cytolytic pathway. Vitronectin is largely expressed in the liver but also in visceral tissue and adrenals. The presence of vitronectin in HDL raises the possibility that certain HDL components can be derived from non-cellular sources or cells distinct from those that synthesise apoA-I in the liver and intestine (Heinecke 2009).

\section{Proteinase Inhibitors}

A family of proteins in HDL contains serine proteinase inhibitor domains (Vaisar et al. 2007). Serine protease inhibitors (serpins) are important regulators of biological pathways involved in inflammation, coagulation, angiogenesis and matrix degradation. HDL-associated serpins are exemplified by alpha-1antitrypsin which in the circulation is exclusively present in HDL (Karlsson et al. 2005; Ortiz-Munoz et al. 2009). Alpha-2-antiplasmin, a serpin that inhibits plasmin and trypsin and inactivates chymotrypsin, is another key proteinase inhibitor which circulates in part associated with HDL. HDL also carries inter-alphatrypsin inhibitor heavy chain $\mathrm{H} 4$ and bikunin, two components of inter-alphatrypsin inhibitors consisting of three of four heavy chains selected from the groups 1 to 4 and one light chain selected from the alpha-1-microglobulin/bikunin precursor or Kunitz-type protease inhibitor 2 groups. The full complex inhibits trypsin, plasmin and lysosomal granulocytic elastase. Inter-alpha-trypsin inhibitor heavy chain $\mathbf{H 4}$ is produced in the liver and is cleaved by kallikrein to yield 100 and $35 \mathrm{kDa}$ fragments in plasma, and the resulting $100 \mathrm{kDa}$ fragment is further converted to a $70 \mathrm{kDa}$ fragment. Bikunin is a light chain of the inter-alpha-trypsin inhibitor, which is produced via proteolytic cleavage of the alpha-1-microglobulin/ bikunin precursor protein together with alpha-1-microglobulin and trypstatin.

Other proteolysis-related proteins which are detected on HDL include haptoglobin-related protein (Hrp), kininogen-1, prothrombin, angiotensinogen and procollagen C-proteinase enhancer-2 (PCPE2). Hrp contains a crippled catalytic triad residue that may allow it to act as a decoy substrate to prevent proteolysis. 
Kininogen-1 (alpha-2-thiol proteinase inhibitor) plays an important role in blood coagulation and inhibits the thrombin- and plasmin-induced aggregation of thrombocytes. Prothrombin is a precursor of thrombin, a key serine protease of the coagulation pathway. Angiotensinogen is a an alpha-2-globulin that is produced constitutively mainly by the liver and represents a substrate for renin whose action forms angiotensin I. PCPE2 binds to the C-terminal propeptide of type I or II procollagens and enhances the cleavage of the propeptide by bone morphogenetic protein 1 (BMP-1, also termed procollagen C-proteinase).

\section{Other Protein Components}

HDL equally transports distinct proteins displaying highly specialised functions. The metabolic purpose of such association is unclear; it might prolong the residence time of a protein or represent a mechanism for protein conservation in the circulation. For example, plasma retinol-binding protein, which delivers retinol from the liver stores to peripheral tissues, co-isolates with HDL3 (Vaisar et al. 2007). In plasma, the complex of retinol-binding protein and retinol interacts with transthyretin, thereby preventing loss of retinol-binding protein by filtration through the kidney glomeruli. As a corollary, transthyretin, a homotetrameric thyroid hormone-binding protein, is equally present on HDL (Hortin et al. 2006; Vaisar et al. 2007; Davidson et al. 2009). Serotransferrin, an iron-transport glycoprotein largely produced in the liver, is also in part associated with HDL. Hemopexin, an iron-binding protein that binds heme and transports it to the liver for breakdown and iron recovery, equally co-isolates with HDL3 (Vaisar et al. 2007).

HDL also carries proteins involved in the regulation of various biological functions, such as Wnt signalling molecules, which participate in cell-to-cell signalling (Neumann et al. 2009), and progranulin, a precursor of granulins which play a role in inflammation, wound repair and tissue remodelling.

In addition, HDL transports lysosomal proteins, such as prenylcysteine oxidase, which is involved in the degradation of prenylated proteins (Vaisar et al. 2007). Other minor abundance proteins reported to be associated with HDL are albumin, alpha-1B-glycoprotein, alpha-amylase, vitamin D-binding protein and platelet basic protein (Vaisar et al. 2007; Davidson et al. 2009).

In addition to proteins, HDL carries a large number of small peptides in the mass range from 1 to $5 \mathrm{kDa}$ (Hortin et al. 2006). These peptides are present in HDL at low concentrations of about $1 \%$ of total HDL protein, with some representing fragments of larger proteins, such as apoB, fibrinogen and transthyretin (Hortin et al. 2006). The association of small peptides with HDL as a vehicle may represent a pathway for peptide delivery or scavenging, in order to slow renal clearance and proteolysis (Hortin et al. 2006).

\section{Heterogeneity in HDL Proteins}

HDL proteins are non-uniformly distributed across HDL subpopulations. Indeed, proteomic analysis of five HDL subpopulations isolated from normolipidemic subjects by isopycnic density gradient ultracentrifugation identifies five distinct 
patterns of distribution of individual protein components across the HDL density subfractions (Davidson et al. 2009) (Fig. 1). The most interesting of these distributions identifies small, dense HDL3b and $3 c$ as particle subpopulations in which seven proteins occur predominantly, notably apoJ, apoL-1, apoF, PON1/3, PLTP and PAF-AH. Activities of HDL-associated enzymes (LCAT, PON1, PAF-AH) are equally elevated in small, dense HDL3c (Kontush et al. 2003; Kontush and Chapman 2010). The HDL3c proteome also contains apoA-I; apoAII; apoD; apoM; SAA 1, 2, and 4; apoC-I; apoC-II; and apoE (Davidson et al. 2009). Consistent with these data, apoL-I (Hajduk et al. 1989), apoF (He et al. 2008; Lagor et al. 2009), apoJ (de Silva et al. 1990a, b; Bergmeier et al. 2004), PON1 (Kontush et al. 2003; Bergmeier et al. 2004), apoA-IV (Bisgaier et al. 1985; Ohta et al. 1985), apoM (Wolfrum et al. 2005), apoD (Campos and McConathy 1986) and SAA1/ 2 (Benditt and Eriksen 1977; Coetzee et al. 1986) are known to preferentially co-isolate with dense HDL3. Furthermore, small, dense HDL may also represent a preferential carrier for human CETP (Marcel et al. 1990). On the other hand, apoE, apoC-I, apoC-II and apoC-III preferentially localise to large, light HDL2 (Schaefer et al. 1979; Cheung and Albers 1982; Schaefer and Asztalos 2007; Davidson et al. 2009) (Fig. 1). Importantly, these associations can in part be confirmed using an alternative approach of gel filtration subfractionation of HDL particles (Gordon et al. 2010).

The low HDL content of the majority of these proteins of less than one copy per HDL particle suggests internal heterogeneity of the HDL3c subfraction. This conclusion is further consistent with the isolation of a unique particle containing the trypanosome lytic factor apoL-I, plus apoA-I and Hrp, in the HDL3 density range (Shiflett et al. 2005).

In a similar fashion, apoF co-isolates with dense HDL (mean density, $1.134 \mathrm{~g} / \mathrm{ml}$ ) which also contains apoA-I, apoC-II, apoE, apoJ, apoD and PON1 (He et al. 2008). Complexes formed by PON1 with human phosphate-binding protein and apoJ represent another example of protein-protein interactions occurring within the HDL particle spectrum (Vaisar 2009).

Specific protein-protein interactions should thus drive the formation of such complexes in the circulation. In support of such a mechanism, PLTP in human plasma resides on lipid-poor complexes dominated by apoJ and proteins implicated in host defence and inflammation (Cheung et al. 2010).

In addition and as mentioned above (Sect. 1), immunoaffinity technique allows separating HDL particles containing only apoA-I (LpA-I) from those containing both apoA-I and apoA-II (LpA-I:A-II) (Duriez and Fruchart 1999). ApoA-I is typically distributed approximately equally between LpA-I and LpA-I:A-II, whereas virtually all apoA-II is in LpA-I:A-II (Duriez and Fruchart 1999). LpA-I and LpA-I:A-II contain approximately 35 and $65 \%$ of plasma apoA-I, respectively (James et al. 1988). On the other hand, approximately half of HDL particles contain apoA-II (Wroblewska et al. 2009). In addition to apoA-II, the two subclasses may differ in their content of other proteins, as exemplified by PON1 which preferably associates with LpA-I (Moren et al. 2008). 


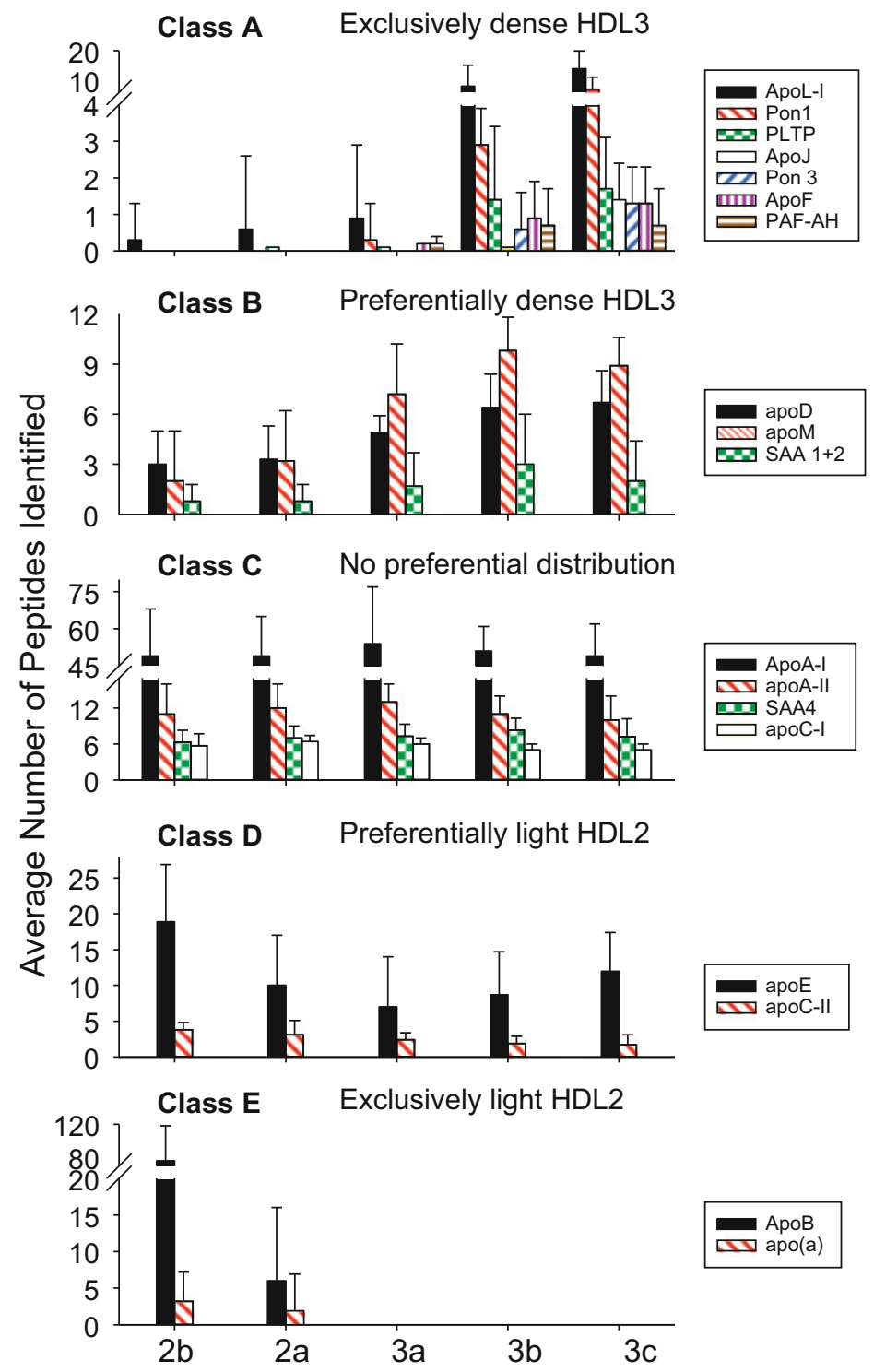

Fig. 1 Abundance pattern of proteins across healthy normolipidemic HDL subpopulations. Class A: exclusively present in small, dense HDL $3 b$ and $3 c$. Class B: enriched in small, dense HDL3b and 3c. Class C: equally abundant across HDL subpopulations. Class D: enriched in large, light HDL2b and 2a. Class E: exclusively present in large, light HDL2b and 2a [modified from (Davidson et al. 2009)] 
LpA-I particles can be further subfractionated according to size. The number of apoA-I molecules in such subpopulations is increased from two to three to four with an increase in the particle size (Gauthamadasa et al. 2010). On the other hand, the entire population of LpA-I:A-II demonstrates the presence of only two apoA-I molecules per particle, while the number of apoA-II molecules varies from one dimeric apoA-II to two and then to three. Upon compositional analyses of individual subpopulations, LpA-I:A-II exhibits comparable proportions for major lipid classes across subfractions, while LpA-I components show significant variability (Gauthamadasa et al. 2010).

Another important subpopulation of HDL particles is formed by apoEcontaining HDL. The presence of apoE facilitates expansion of the lipid core as a result of the accumulation of cholesteryl ester, with formation of large, lipid-rich HDL; these particles represent an excellent ligand for the LDL receptor (Hatters et al. 2006).

The diversity of molecules which bind to HDL suggests that the lipoprotein can serve as a versatile adsorptive surface for proteins and peptides to form complexes playing roles not only in lipid metabolism but equally in acute-phase response, innate immune response, complement activation, plaque stability and proteolysis inhibition (Heinecke 2009). As the abundance of the most of HDL-associated proteins is below $1 \mathrm{~mol} / \mathrm{mol}$ HDL (i.e. less than 1 copy per HDL particle), it remains however unclear as to how they are distributed among minor HDL subpopulations of potentially distinct origin and function. Specific proteins may therefore be confined to distinct HDL subpopulations of distinct origin and function, which are differentially distributed across the HDL particle spectrum (Davidson et al. 2009). The HDL fraction as a whole therefore appears to represent "a collection of individualised species with distinct functionalities that happen to have similar physicochemical properties" (Shah et al. 2013) and are primarily defined by specific protein-protein interactions facilitated by the presence of phospholipid, rather than "a transient ensemble of randomly exchanging proteins" (Gordon et al. 2013; Shah et al. 2013).

\subsubsection{Protein Isoforms, Translational and Posttranslational Modifications}

In line with the evolvement of proteomics, a large number of proteins have been identified in HDL as described in the previous section (Vaisar et al. 2007). In addition, most proteins are expressed as different isoforms due to co- and posttranslational modifications (Karlsson et al. 2005; Candiano et al. 2008). This makes the proteome of HDL both complex and dynamic, which most likely result in various HDL particles with different protein composition in respect to the environment. Common posttranslational modifications (PTMs) such as glycosylations, truncations and phosphorylations change the charge and/or the size of the protein, which can be utilised for separation of the isoforms, and modern mass spectrometric techniques can be used to detect, characterise and nowadays also measure even small mass differences in proteins. However, although isoforms of major HDL proteins have been known for decades (Zannis et al. 1980; Hussain and Zannis 1990), surprisingly 
little is still known on how these variations of the HDL proteome affect the functionality. The following is a comprehensive review of isoforms patterns described in common human HDL apolipoproteins, apoA-I, apoA-II, apoC-III and SAA, and their possible functional relevance.

ApoA-I ( $\mathrm{p} I 5.3 / 28 \mathrm{kDa}$, accession no. P02647) is normally found as different charge isoforms; besides the major isoform (70-75\% of total apoA-I) and the slightly more basic pre-apoA-I (5-10\% of total apoA-I), also two more acidic isoforms are generally detected by isoelectric focusing (IEF) and 2D gel electrophoresis (2-DE) (Contiero et al. 1997; Karlsson et al. 2005). The nature of these acidic isoforms is still unclear. An early report suggested deamidation of Gln or Asn residues, resulting in a +1 charge shift, which could be formed during the analytical procedure (Ghiselli et al. 1985). At the same time, a few reports indicated the importance of acidic apoA-I in vivo; increased levels of acidic apoA-I, while decreased levels of the major form, were found in LDL from obese subjects, especially in women (Karlsson et al. 2009). Also, higher degree of deamidated apoA-I has been shown in relation to diabetes (Jaleel et al. 2010) and acidic apoA-I may be more vulnerable to methionine oxidation (Fernandez-Irigoyen et al. 2005). In 2-DE HDL protein patterns also 30-35 $\mathrm{kDa}$ variants of apoA-I are usually detected (Karlsson et al. 2005). Although mass spectrometry (MS) analysis was in agreement with O-glycosylation at two potential sites (Thr78 or Thr92), this has not been confirmed by others, and it is generally regarded that apoA-I is not N-linked or O-linked glycosylated. In contrast, non-enzymatically glycation of apoA-I has been found in association to diabetes and believed to affect apoA-I functions, such as LCAT activation (Fievet et al. 1995; Nobecourt et al. 2007; Park et al. 2010).

Another potentially important PTM of apoA-I is truncation. During atherosclerotic inflammation, apoA-I might be $\mathrm{N}$-terminally and C-terminally truncated by released proteases. Specific cleavage sites at Tyr42, Phe57, Tyr216 and Phe253 for chymase have been identified that in reconstituted HDL reduces its ability to promote cholesterol efflux (Lee et al. 2003; Usami et al. 2013). Low amounts of C-terminally truncated apoA-I can be measured in normal serum (Usami et al. 2011) and fragmented apoA-I is a feature in plasma from children with nephrotic syndrome, a condition linked to higher risk of atherosclerosis (Santucci et al. 2011). Truncation has also been implicated in apoA-I dimerisation as studied in apoA-I Milano (R197C) and apoA-I Paris (R175C) (Calabresi et al. 2001; Favari et al. 2007; Gursky et al. 2013). Notably, apoA-I with an apparent molecular mass of $50 \mathrm{kDa}$, consistent with dimeric apoA-I, has been found in patients with myocardial infarction (Majek et al. 2011) but also appears to be present in HDL from healthy individuals (Karlsson et al. 2005). Finally, oxidatively modified apoA-I have been extensively studied during the recent years by the help of MS techniques, as described in detail in several reviews elsewhere (e.g. Nicholls and Hazen 2009; Shao 2012). It has been proposed that myeloperoxidase-mediated inflammation results in oxidation of apoA-I. Specific sites have been identified for methionine oxidation, for nitrated/chlorinated tyrosines and for lysines modified by reactive carbonyls. Importantly, the modifications have been coupled to 
functional impairment in apoA-I activity such as ABCA1-mediated cholesterol efflux and are linked to cardiovascular disease.

ApoA-II (pI 5.0/8.7 kDa, accession no. P02652) is mostly found as two isoforms in HDL that differ slightly according to $\mathrm{p} I$ and molecular mass, probably due to O-linked glycosylation/sialylation (Karlsson et al. 2005; Halim et al. 2013). Similar to apoA-I, the protein is produced as a more basic pro-form (Hussain and Zannis 1990). In contrast, apoA-II appears to be quickly processed to the mature form, as the pro-form is not found in the circulation. In addition to glycosylation, phosphorylation at Ser68, C-terminal truncated variants (des-Gln and des-Thr-Gln) and a cysteinylated variant has been detected in the circulation (Jin and Manabe 2005; Nelsestuen et al. 2008; Zhou et al. 2009). ApoA-II also forms a homodimer at Cys29 that is abundant in plasma (Gillard et al. 2005; Jin and Manabe 2005). Overall, more than ten different variants of apoA-II are present in humans, but the physiological relevance of this heterogeneity is unclear. However, sialylated apoA-II appear to be selectively associated to HDL3 (Remaley et al. 1993; Karlsson et al. 2005), and elevated levels of modified apoA-II isoforms have been linked to premature delivery in pregnant women (Flood-Nichols et al. 2013).

ApoC-III ( $\mathrm{p} I$ 4.7/8.8 $\mathrm{kDa}$, accession no. P02656) is generally found as three charge isoforms depending on O-linked glycosylation (GalGalNAc) at Thr94 with or without sialylation; disialylated apoC-III 2 , monosialylated apoCIII 1 and non-sialylated apoC-III ${ }_{0}$ (Karlsson et al. 2005; Bruneel et al. 2008). An early report showed that glycosylation is not necessary for apoC-III secretion and does not affect its relative affinity to different lipoprotein particles (Roghani and Zannis 1988), and, as judged by gel electrophoresis and MS analysis of HDL and plasma, the non-sialylated variant is least abundant, usually less than $5 \%$ of total apoC-III in normal individuals (Wopereis et al. 2003; Bruneel et al. 2008; Mazur et al. 2010; Holleboom et al. 2011). In addition to glycosylation, apoC-III can also be C-terminal truncated (des-Ala and des-Ala-Ala), which further increases the number of isoforms (Bondarenko et al. 1999; Jin and Manabe 2005; Nicolardi et al. 2013a). Interestingly, novel results strongly suggest that glycosylation of apoC-III is an important event in the regulation of lipid metabolism (Holleboom et al. 2011; Baenziger 2012). Thus, apoC-III is exclusively glycosylated by GalNAc transferase 2 (GALNT2) (Holleboom et al. 2011; Schjoldager et al. 2012), and heterozygotes with a loss-of-function mutation in GALNT2 present with an altered apoC-III isoform pattern with more of the non-sialylated variant and less of the monosialylated variant, while the total apoC-III plasma concentration was about the same as compared to wild-type controls (Holleboom et al. 2011). This is then linked to reduced inhibition of lipoprotein lipase and improved triglyceride clearance. In line, the production rate of apoC- $-\mathrm{III}_{1}$ and $-\mathrm{III}_{2}$ is more strongly correlated with

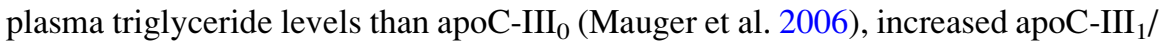
apoC- III $_{0}$ ratio has been found in diabetic subjects (Jian et al. 2013), and HDL3 from subjects with low HDL-C is characterised by higher levels of monosialylated apoC-III than subjects with high HDL-C (Mazur et al. 2010). The evaluation of apoC-III isoforms is complicated by the fact that apoC-III 0 can be separated into a non-glycosylated form and glycosylated but non-sialylated forms (Bruneel 
et al. 2008; Holleboom et al. 2011; Nicolardi et al. 2013a). Moreover, a recent MS study of 96 serum samples showed that $30 \%$ of the individuals displayed an apoCIII pattern with additional glycosylated variants, characterised by fucosylation (Nicolardi et al. 2013b). The relevance of these glycosylated non-sialylated variants of apoC-III, as of the C-terminal truncated forms, is yet unclear, but may explain somewhat contradictory results showing higher relative levels of non- and lesssialylated apoC-III in obese subjects than in lean subjects (Harvey et al. 2009; Karlsson et al. 2009), although obesity is generally associated with high triglyceride levels.

SAA exists in a form of SAA1 (pI 5.9/11.7 kDa, accession no. P0DJI8) and SAA2 (pI 8.3/11.6 kDa, accession no. P0DJI9). The two proteins display about $93 \%$ sequence homology, and depending on natural variation in alleles, SAA1 is separated into five isoforms, SAA1.1 to SAA1.5, and SAA2 is separated into two isoforms SAA2.1 and SAA2.2 (often also denoted as alpha, beta, etc.), with one to three amino acid difference between the isoforms. In addition, both $\mathrm{N}$-terminal and C-terminal truncated variants of SAA1/SAA2 are detected in serum (Ducret et al. 1996; Kiernan et al. 2003; de Seny et al. 2008). By using a combined 2-DE/ MS and SELDI-TOF approach, eight isoforms were identified in HDL after LPS infusion in healthy individuals; besides native SAA1.1 and SAA2.1, N-terminal truncations (des-R, -RS and -RSFF) of each variant were also found (Levels et al. 2011). Interestingly, a subgroup, based on HDL protein profile, characterised by elevated antioxidative PON1 activity showed a delayed response of SAA to LPS in particular for the most truncated (des-RSFF) variants. Otherwise, very little is known about differential physiological relevance of the SAA isoforms. However, SAA2.1 but not SAA1.1 has been shown to promote cholesterol efflux from macrophages (Kisilevsky and Tam 2003). Today, contradictory results make it unclear whether the increased level of SAA in inflammation, believed to replace apoA-I in HDL, actually is a mechanism in atherosclerosis or merely is a marker for inflammation (de Beer et al. 2010; Chiba et al. 2011; Kisilevsky and Manley 2012). Future differential quantitative MS analysis of the highly homologous SAA isoforms such as described by Sung et al. (2012) may resolve this controversy.

Sensitive MS techniques have revealed a large number of proteins associated to HDL. Most of them are also expressed as different isoforms depending on translational and posttranslational modifications. This leads to a need to develop MS-based methods for specific and reliable quantification of protein isoforms. With appropriate standards, measurements can be performed with low coefficient of variation and with a specificity superior to, e.g. immunoassays. Consequently, such applications in the field of HDL are being presented by using, e.g. multiple reaction monitoring and top-down proteomics with high-resolution MS (Mazur et al. 2010; Sung et al. 2012). Another interesting and fairly simple approach is to use ratio determinations, e.g. modified/native protein expression (Nelsestuen et al. 2008). As these measures are concentration-independent, they bear a potential to reduce individual variations. Furthermore, such MS approaches are not only useful for PTMs but also of value to understand the impact of protein variations caused by genetic polymorphism. For example, a recent study of heterozygotes 
with an apoA-I mutation (K131Del) showed that, in contrast to what could be expected, the mutant protein was more abundantly expressed in HDL than the native protein (Ljunggren et al. 2013). Herein four HDL proteins that are all expressed as different isoforms have been discussed: two (apoA-I and apoC-III) in which PTMs have been shown to be important for lipid metabolism and two (apoA-II and SAA) in which the role of PTMs is still unclear. In light of the vital importance of carboxylations and truncations in the processes of haemostasis, which also involves other PTMs such as phosphorylation, hydroxylation, glycosylation and sulphation, it appears highly unlikely that the diversity in the "HDLome" would not be relevant for lipid metabolism and cardiovascular disease. Therefore, characterisation of PTMs is probably one of the most challenging but also one of the most important tasks in order to understand the complex function of HDL.

\section{$2.2 \quad$ Lipidome}

The real power of lipidomic technologies involving mass spectrometry results from their ability to provide quantitative data on individual molecular species of lipids and on low-abundance lipid molecules. The pioneering study of Wiesner and colleagues published in 2009 (Wiesner et al. 2009) provided reference values for the lipidome of HDL isolated from healthy normolipidemic controls by FPLC. In an attempt to further characterise HDL composition and address its inherent heterogeneity, we recently reported the phospho- and sphingolipidome of five major HDL subpopulations isolated from healthy normolipidemic subjects (Camont et al. 2013).

\subsubsection{Phospholipids}

Phosphatidylcholine is the principal plasma phospholipid that accounts for 32 $35 \mathrm{~mol} \%$ of total lipids in HDL (Wiesner et al. 2009) (Table 3). PC is a structural lipid, consistent with its even distribution across HDL subpopulations (Fig. 2). Major molecular species of PC are represented by the 16:0/18:2, 18:0/18;2 and 16:0/20:4 species (Lhomme et al. 2012). As compared to other lipoproteins, HDL is enriched in PC containing polyunsaturated fatty acid moieties (Wiesner et al. 2009).

LysoPC is an important phospholipid subclass in HDL (1.4-8.1 mol \% of total lipids; Table 3). It is derived from the regulated degradation of PC by phospholipases, including LCAT, consistent with the preferential association of the latter with HDL particles (Kontush et al. 2007). More specifically, LCAT was reported earlier to associate mainly with small, dense HDL particles, which are also enriched in lysoPC by approximately twofold as compared to large, light HDL (Camont et al. 2013) (Fig. 2). LysoPC is also produced by the hydrolytic action of Lp-PLA $A_{2}$ on oxidised PC or by secreted $\mathrm{PLA}_{2}$ under pro-atherogenic conditions, such as oxidative stress and inflammation, and constitutes therefore a potential biomarker of inflammation. Major molecular species of HDL lysoPC contain saturated fatty acid moieties of predominantly 16 and 18 carbon atoms, reflecting LCAT preference for 16 and 18 carbon atom long PCs (Lhomme et al. 2012). As 
Table 3 Major components of the HDL lipidome

\begin{tabular}{|c|c|}
\hline Lipid class & HDL content in mol $\%$ of total lipids \\
\hline Phospholipids & $37.4-49.3$ \\
\hline Phosphatidylcholine & $32-35$ \\
\hline PC-plasmalogen & $2.2-3.5$ \\
\hline LysoPC & $1.4-8.1$ \\
\hline Phosphatidylethanolamine & $0.70-0.87$ \\
\hline PE-plasmalogen & $0.54-0.87$ \\
\hline Phosphatidylinositol & $0.47-0.76$ \\
\hline Cardiolipin & $0.077-0.201$ \\
\hline Phosphatidylserine & $0.016-0.030$ \\
\hline Phosphatidylglycerol & $0.004-0.006$ \\
\hline Phosphatidic acid & $0.006-0.009$ \\
\hline Sphingolipids & $5.7-6.9$ \\
\hline Sphingomyelin & $5.6-6.6$ \\
\hline Ceramide & $0.022-0.097$ \\
\hline Hexosyl Cer & $0.075-0.123$ \\
\hline Lactosyl Cer & $0.037-0.060$ \\
\hline S1P d18:1 & $0.015-0.046$ \\
\hline S1P d18:0 & 0.007 \\
\hline SPC d18:1 & 0.001 \\
\hline Neutral lipids & $46.7-54.0$ \\
\hline Cholesteryl esters & $35-37$ \\
\hline Free cholesterol & $8.7-13.5$ \\
\hline Triacylglycerides & $2.8-3.2$ \\
\hline Diacylglycerides & $0.17-0.28$ \\
\hline \multicolumn{2}{|l|}{ Minor lipids } \\
\hline Free fatty acids & $16: 0,18: 0,18: 1^{\mathrm{a}}$ \\
\hline Isoprostane-containing PC & ND (IPGE2/D2-PC (36:4)) ${ }^{\mathrm{a}}$ \\
\hline
\end{tabular}

Data are shown for HDL obtained from normolipidemic healthy subjects according to Deguchi et al. (2000), Kontush et al. (2007), Wiesner et al. (2009), Camont et al. (2013), Stahlman et al. (2013), Pruzanski et al. (2000), Sattler et al. (2010), Argraves et al. (2011)

$S P C$ sphingosylphosphorylcholine, SIP sphingosine-1-phosphate, IPGE2 isoprostaglandin E2 ${ }^{a}$ no quantitative data available, major molecular species identified

considerable amounts of serum lysoPC are also associated with albumin (Wiesner et al. 2009), HDL contamination by the both compounds is typical for FPLC isolation. However, in HDL isolated by isopycnic density gradient ultracentrifugation, lysoPC content in HDL is two- to tenfold lower (Camont et al. 2013; Stahlman et al. 2013).

Phosphatidylethanolamine (PE) is moderately abundant in HDL (0.7$0.9 \mathrm{~mol} \%$ of total lipids; Table 3), and its content tends to increase with increasing HDL hydrated density (Wiesner et al. 2009; Camont et al. 2013) (Fig. 2). PE principal molecular species are represented by the $36: 2$ and 38:4 fatty acid residues in HDL (Kontush et al. 2007). 


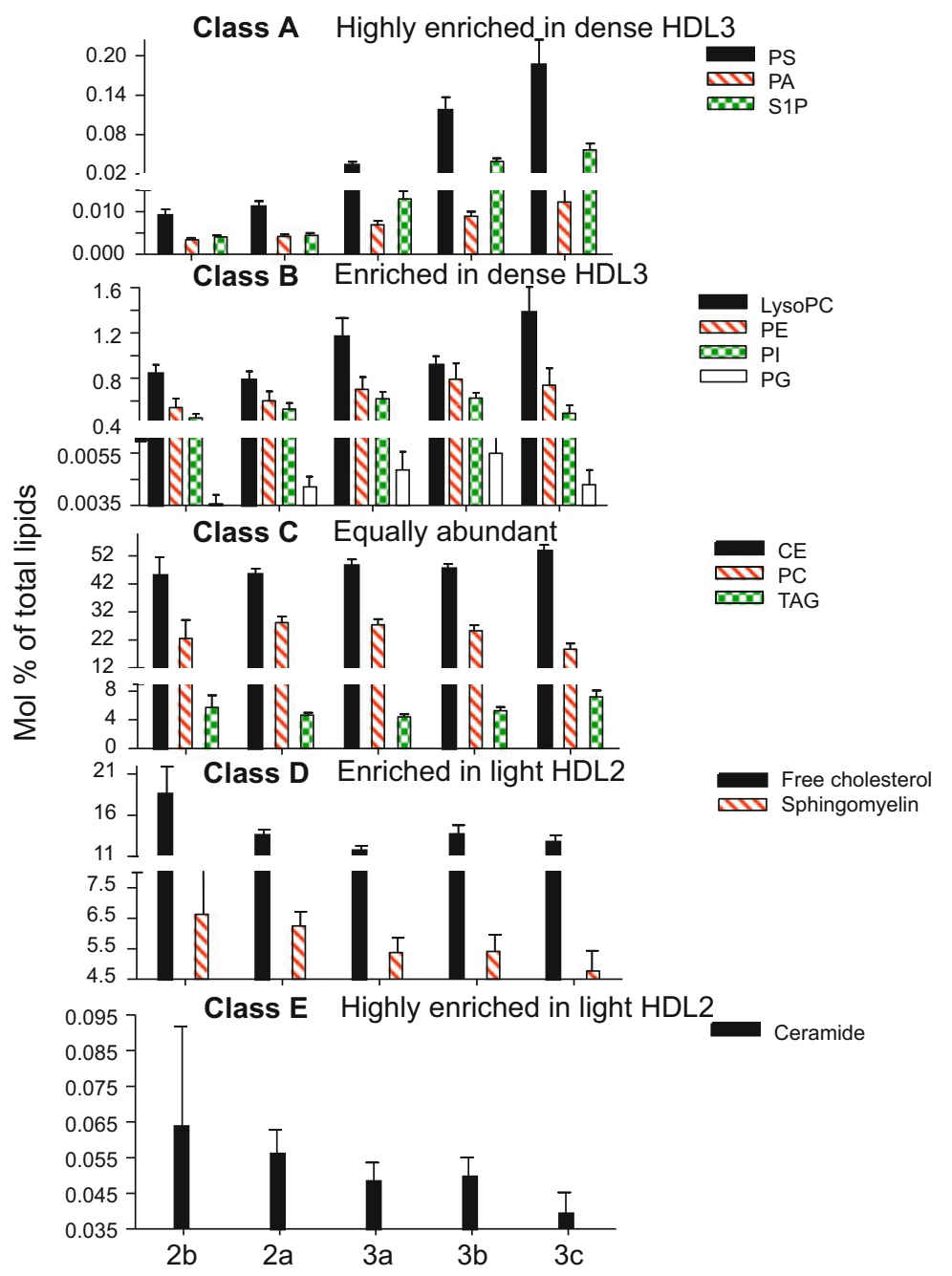

Fig. 2 Abundance pattern of lipids across healthy normolipidemic HDL subpopulations. Class A: highly enriched in small, dense HDL3b and 3c ( $>1.5$-fold relative to HDL2b). Class B: enriched in small, dense HDL3b and 3c (1.2-1.5-fold relative to HDL2b). Class C: equally abundant across HDL subpopulations ( $<1.2$-fold variations between HDL2b and HDL3b $+3 c)$. Class D: enriched in large, light HDL2b $(1.2-1.5$-fold relative to HDL3b $+3 c)$. Class E: highly enriched in large, light HDL2b ( $>1.5$-fold relative to HDL3b $+3 c)$. S1P, sphingosine-1-phosphate

Plasmalogens contain a vinyl ether-linked fatty acid essential for their specific antioxidative properties (Maeba and Ueta 2003). PC-plasmalogens are the most abundant species in HDL (2.2-3.5 mol \%) but represent less than $10 \%$ of total PC (Stahlman et al. 2013). On the contrary, PE-plasmalogens and PE are equally abundant in HDL (0.6-0.9 mol \%; Table 3). PC- and PE-plasmalogens contain 
mainly polyunsaturated species: $38: 5$ and 36:2 in PC-plasmalogens (Stahlman et al. 2013) and 18:0/20:4 and 16:0/20:4 in PE-plasmalogens (Wiesner et al. 2009).

Phosphatidylinositol (PI), phosphatidylserine (PS), phosphatidylglycerol (PG), phosphatidic acid (PA) and cardiolipin are negatively charged phospholipids present in HDL (Table 3) which may significantly impact on its net surface charge (Rosenson et al. 2011; Lhomme et al. 2012). The content of these lipids can thereby modulate lipoprotein interactions with lipases, membrane proteins, extracellular matrix and other protein components; indeed, such interactions are largely charge-dependent.

PI, similarly to PE, is moderately abundant in HDL ( $0.5-0.8 \mathrm{~mol} \%$; Table 3$)$ and tends to be enriched in small, dense HDL (Fig. 2). Major molecular species of PI in HDL include the 18:0/20:3 and 18:0/20:4 species (Lee et al. 2010).

PS is a minor negatively charged phospholipid component of HDL $(0.016$ $0.030 \mathrm{~mol} \%$; Table 3 ). This phospholipid was very recently reported to be highly enriched (34-fold) in the small, dense HDL3c subpopulation relative to large, light HDL2 (Fig. 2) (Camont et al. 2013) as well as in small discoid pre $\beta$ HDL and small nascent HDL formed by ABCA1 (up to $2.5 \mathrm{~mol} \%$ of total lipids) (see Kontush and Chapman 2012 for review). Interestingly, small, dense HDL also displayed potent biological activities which correlated positively with PS content in HDL (Camont et al. 2013). This lipid could therefore, in part, account for enhanced functionality of HDL3c.

PA, a second messenger, is both a common metabolic precursor and an enzymatic product of phospholipid metabolism. This negatively charged lipid is present in very low abundance in HDL ( $0.006-0.009 \mathrm{~mol} \%$; Table 3$)$ but, similarly to PS, is enriched in small, dense HDL (by more than threefold) (Camont et al. 2013) (Table 3). This observation might reflect preferential association of PA with apoL-I which is equally enriched in small, dense HDL (Kontush and Chapman 2012).

PG is a metabolic precursor of cardiolipin present in HDL in very low amounts (0.004-0.006 mol \%; Table 3). PG tends to be enriched in small, dense particles (Camont et al. 2013) (Fig. 2).

Cardiolipin is a minor anionic phospholipid present in trace amounts in HDL (0.08-0.2 mol \%; Table 3). This lipid with potent anticoagulant properties may contribute to the effects of lipoproteins on coagulation and platelet aggregation (Deguchi et al. 2000).

Together, these data indicate that although negatively charged lipids represent minor HDL constituents ( $0.8 \mathrm{~mol} \%$ of total lipids), they are highly enriched in small, dense HDL, consistent with the elevated surface electronegativity of this subpopulation (Rosenson et al. 2011).

Isoprostanes are well established as biomarkers of oxidative stress and are predominantly associated with HDL (see Kontush and Chapman 2012 for review). Major molecular species of isoprostane-containing PCs include 5,6-epoxyisoprostaglandine A2-PC (EIPGA2-PC) 36:3, 5,6 EIPGE2-PC 36:4, IPGE2/D2PC 36:4, IPGF-PC 36:4, IPGE2/D2-PC 38:4 and IPGF-PC 38:4 (Pruzanski et al. 2000) (Table 3). 


\subsubsection{Sphingolipids}

Sphingomyelin, a structural lipid which enhances surface lipid rigidity (Rye et al. 1996; Saito et al. 2000), is the major sphingolipid in circulating HDL (5.6$6.6 \mathrm{~mol} \%$ of total lipids) (Wiesner et al. 2009; Camont et al. 2013; Stahlman et al. 2013) (Table 3), which largely originates from triacylglyceride-rich lipoproteins and only to a minor extent from nascent HDL (Nilsson and Duan 2006). Major molecular species of sphingomyelin are the 16:0 and 24:1 species (Lhomme et al. 2012). Unlike negatively charged PL, sphingomyelin is depleted by up to $30 \%$ in small, dense relative to large, light HDL (Kontush et al. 2007; Camont et al. 2013) (Fig. 2). This result may, in part, reflect the low abundance of sphingomyelin in nascent HDL, a metabolic precursor of HDL3c, and suggest distinct metabolic pathways for HDL subpopulations.

Among lysosphingolipids, S1P is particularly interesting as this bioactive lipid plays key roles in vascular biology (Lucke and Levkau 2010). More than $90 \%$ of circulating sphingoid base phosphates are found in HDL and albumin-containing fractions (Table 3) (Kontush and Chapman 2012). Interestingly, S1P associates preferentially with small, dense HDL particles (up to tenfold enrichment compared to large, light HDL) (Kontush et al. 2007) (Fig. 2) consistent with the high content in apoM, a specific carrier of S1P, in small, dense particles (Davidson et al. 2009). Other biologically active lysosphingolipids carried by HDL are represented by lysosphingomyelin and lysosulfatide (Lhomme et al. 2012).

Ceramide is a sphingolipid intermediate implicated in cell signalling, apoptosis, inflammatory responses, mitochondrial function and insulin sensitivity (Lipina and Hundal 2011). This lipid is poorly transported by HDL, which carries only $25 \mathrm{~mol} \%$ of total plasma ceramide (Wiesner et al. 2009), and constitutes only between 0.022 and $0.097 \mathrm{~mol} \%$ of total HDL lipids (Wiesner et al. 2009; Argraves et al. 2011; Camont et al. 2013; Stahlman et al. 2013) (Table 3). Similarly to sphingomyelin, this product of sphingomyelin hydrolysis is enriched in large, light HDL (Fig. 2), suggesting common metabolic pathways for these lipids. This hypothesis is however not supported by the pattern of major molecular species of ceramide observed in HDL, which are the 24:0 and 24:1 species (Wiesner et al. 2009; Stahlman et al. 2013).

Lipidomic data on glycosphingolipids, gangliosides and sulfatides are scarce (Lhomme et al. 2012). Hexosyl and lactosyl species constitute the major glycosphingolipids in plasma lipoproteins (Scherer et al. 2010) (Table 3).

\subsubsection{Neutral Lipids}

Unesterified (free) sterols are located in the surface lipid monolayer of HDL particles and regulate its fluidity. HDL sterols are dominated by cholesterol, reflecting the key role of lipoproteins in cholesterol transport through the body. Other sterols are present in lipoproteins at much lower levels as exemplified by minor amounts of lathosterol, ergosterol, phytosterols ( $\beta$-sitosterol, campesterol), oxysterols and estrogens (largely circulating as esters) (Kontush and Chapman 2012). Free cholesterol, whose affinity for sphingomyelin is now well established, tends to preferentially associate with large, light HDL (Fig. 2). 
Cholesteryl esters (CE) are largely (up to $80 \%$ ) formed in plasma HDL (Fig. 2), as a result of transesterification of PL and cholesterol catalysed by LCAT. These highly hydrophobic lipids form the lipid core of HDL and contribute up to $36 \mathrm{~mol} \%$ of total HDL lipid (Wiesner et al. 2009; Camont et al. 2013; Stahlman et al. 2013) (Table 3). Most of HDL CE is accounted for by cholesteryl linoleate (Lhomme et al. 2012). A pioneering work on CE molecular species distribution across HDL subpopulations using gas chromatography showed very similar profiles between HDL2 and HDL3 particles (Vieu et al. 1996).

HDL-associated triacylglycerides (TAG) are dominated by species containing oleic, palmitic and linoleic acid moieties (Lhomme et al. 2012) and represent around $3 \mathrm{~mol} \%$ of total HDL lipids (Vieu et al. 1996; Kontush et al. 2007; Wiesner et al. 2009; Camont et al. 2013; Stahlman et al. 2013) (Table 3). Similarly to CE, TAG species profile is conserved between HDL2 and HDL3 (Vieu et al. 1996).

Minor bioactive lipids present in HDL include diacylglycerides (DAG), monoacylglycerides (MAG) and free fatty acids (Lhomme et al. 2012). In the study of Vieu et al., sn-1,2 and sn-1,3 isomers of DAG species were identified in HDL (Vieu et al. 1996). More recently, detailed characterisation of DAG molecular species in HDL revealed 16:0/18:1 and 18:1/18:1 as major species, estimating their content at $0.2 \mathrm{~mol} \%$ of total lipids (Stahlman et al. 2013) (Table 3).

HDL composition in free fatty acids shows a predominance of palmitic, stearic and oleic acid-containing species (Lhomme et al. 2012).

Together, available lipidomic studies have already provided detailed characterisation of HDL isolated using two complementary techniques, FPLC (Wiesner et al. 2009) and ultracentrifugation (Kontush et al. 2007; Camont et al. 2013). The latter studies demonstrated preferential association of negatively charged lipids (PS, PA) and S1P with small, dense HDL together with preferential association of sphingomyelin and ceramide with large, light particles, consistent with distinct metabolic origins and potent biological activities of small, dense HDL (Camont et al. 2013). These data illustrate the power of lipidomics to provide crucial information on the metabolism and function of lipoproteins relevant for the development of cardiovascular disease, which can in turn deliver novel biomarkers of cardiovascular risk.

\section{The Structure of HDL}

\subsection{Introduction/Brief History}

When it was becoming clear that plasma levels of HDL were inversely correlated with cardiovascular disease, numerous laboratories were already doing pioneering work on the structure of human plasma HDL (Edelstein et al. 1972; Jonas 1972; Laggner et al. 1973; Atkinson et al. 1974; Schonfeld et al. 1976; Tardieu et al. 1976). Knowing little about the nature of the stabilising proteins, Scanu and colleagues used detailed compositional studies to build models of human HDL3 
particles (Shen et al. 1977; Scanu 1978). Concepts derived from these studies with respect to fundamental packing of the protein and various lipid components still apply today. After the sequences of the major HDL proteins, apolipoprotein A-I and apoA-II, were reported in the 1970s (Brewer et al. 1972, 1978), Segrest (Segrest et al. 1974) and others (McLachlan 1977) quickly noted periodically repeating units that, when mapped on a helical wheel plot, indicated the presence of amphipathic alpha helices. With hydrophobic faces mediating lipid interactions and polar faces interacting with water, these structures turned out to be responsible for the detergent-like ability of these proteins to solubilise lipids into stabile lipoprotein particles. At around the same time, Jonas and colleagues were learning how to combine purified apolipoproteins with lipids under control of detergents to produce recombinant forms of HDL (Matz and Jonas 1982). Furthermore, the work of Nichols et al was exploring new ways to characterise these particles by native electrophoresis (Nichols et al. 1983). Because the electron microscopy work of Forte indicated that they likely have a discoid shape (Forte and Nordhausen 1986), these particles were referred to as reconstituted (r)HDL discs. Their compositional simplicity and homogeneity quickly made them a mainstay for HDL structural studies.

Segrest et al. and others (Wlodawer et al. 1979) proposed in the late 1970s that the $\alpha$-helices of apoA-I were arranged around the circumference of discoidal HDL with the long axis of the helices perpendicular to the acyl chains (Fig. 3). This became known as the "belt" or "bicycle wheel" model. Alternatively, other investigators theorised that the 22-amino acid helical repeats were an ideal length to traverse the bilayer edge with the helices parallel to the acyl chains (Jonas et al. 1989; Nolte and Atkinson 1992). The intervening proline residues were thought to induce hairpin turns that punctuated the repeats. This "picket fence" model was favoured during the early 1990s because of supporting infrared (IR) spectroscopy studies (Brasseur et al. 1990) and the fact that the model accounted nicely for the experimentally observed size classes of apoA-I containing HDL (Wald et al. 1990). Furthermore, the plausibility of the picket fence model was supported by Phillips et al. using molecular modelling techniques (Phillips et al. 1997).

However, the publication of the first successful X-ray crystal structure of a lipid-free fragment of apoA-I by Borhani et al. (1997) brought fresh energy to the debate. The crystal structure showed a tetramer of highly $\alpha$-helical apoA-I molecules arranged in a ring-shaped complex with no evidence of hairpin turns that would be prevalent in the picket fence. The belt model picked up more support with methodologically updated IR experiments performed by Koppaka et al. (1999) that contradicted the earlier IR studies supporting the picket fence in rHDL discs. Over the next few years, several laboratories reported results that supported the belt orientation using methodologies like fluorescence energy transfer (Li et al. 2000) and lipid-based fluorescence quenching (Panagotopulos et al. 2001). With the question of helical orientation largely addressed, much of the focus for the first decade of the 2000s centred on determining the spatial relationships between 
a

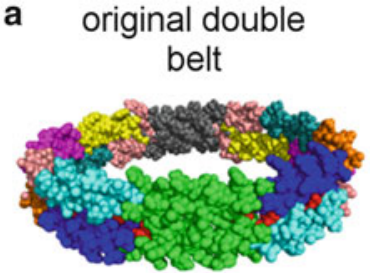

b belt and buckle

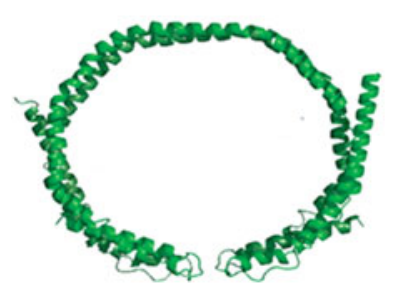

c looped belt
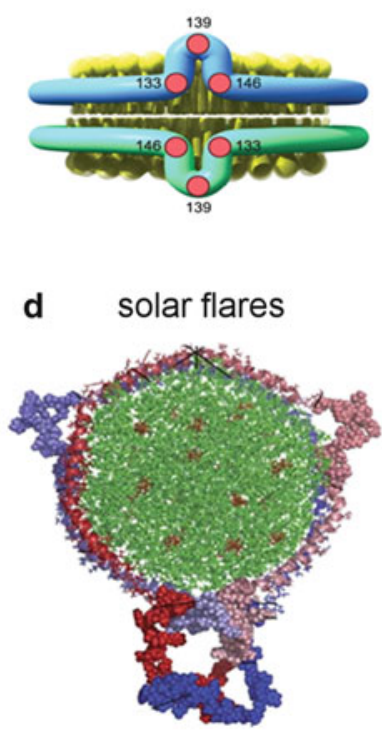

e double super helix

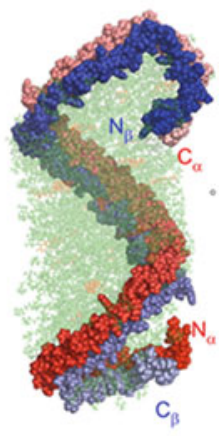

Fig. 3 The double belt model for a rHDL discoidal particle and its various refinements. The classic LL 5/5 double belt model for apoA-I as proposed by Segrest (Segrest et al. 2000) is shown in panel (a). Each amphipathic helical domain is shown in a different colour with helix 5 in green at the front. This model did not include the N-terminal 43 a.a. The "belt and buckle" model (b) does not form a continuous circle around the particle but has its N-terminal 43 a.a. folding back across the belts (40). The "looped belt" (c) features a localised separation between residues 133 and 146 (helix 5) (41). The "solar flares" model (d) encapsulates the lipid with an antiparallel double belt but has disorganised domains that resemble solar flares erupting between residues 165 and 180. The double superhelix model (e) maintains similar intermolecular contact among the molecules of apoA-I as the double belt, but the lipid is stabilised as an elongated micelle and no intramolecular contact is made between the $\mathrm{N}$ - and C-termini of apoA-I

apoA-I molecules on these discs. This effort was aided by the detailed "double belt" computer model put forth in the classic paper by Segrest (Segrest et al. 1999).

The preceding paragraphs quickly summarise a tremendous amount of work between 1970 and 2000 aimed at understanding the structure of HDL and its recombinant forms, and by no means does it do justice to all contributions. For more complete discussions of this period of HDL structural research, the reader is referred to the classic review series by Brouillette (Brouillette and Anantharamaiah 1995; Brouillette et al. 2001). In addition, due to space limitations, this section will not focus on the structure of lipid-free apolipoproteins nor their proposed mechanisms for lipid binding and particle generation. The reader is directed to the recent review by Phillips (Phillips 2013) for an excellent discussion of these issues. For the purposes of this section, we will focus on work done over the last 14 years that has built upon the concept of the double belt model in both reconstituted and "real" circulating plasma HDL. 


\subsection{HDL in the Test Tube}

\subsubsection{Discoid HDL}

Discoid HDLs probably exist in human plasma but are extremely short-lived due to the fact that they are excellent substrates for LCAT (Jonas 2002). HDL discs have been detected in sequestered compartments like peripheral lymph and interstitial fluid which likely have lower LCAT activity (Sloop et al. 1983). As stated above, discs can be reconstituted in vitro, and these particles exhibit many of the traits of native HDL such as LCAT activation (Jonas and McHugh 1984), lipid transfer (Davidson et al. 1995) and receptor binding (Liadaki et al. 2000).

The most well-studied rHDL discs consist of two apoA-I molecules, are $96 \AA$ in diameter and $\sim 47 \AA$ in thickness and contain 150-200 phospholipid molecules (Jonas 1986; Jonas et al. 1989). This "benchmark" particle was used as a basis for the original double belt molecular model (Segrest et al. 1999). In this model, two ring-shaped apoA-I molecules encapsulate a lipid membrane leaflet in an antiparallel orientation (Segrest 1977; Segrest et al. 1999; Brouillette et al. 2001; Klon et al. 2002a) in which helix 5 of both apoA-I molecules directly oppose each other (Fig. 3a). In this configuration, there are two possible interfaces between the molecules depending on how they are stacked: left to left (LL) and right to right (RR). Computer analysis indicated that an LL interface in which helix 5 (more specifically, glycine 129) of both molecules are directly opposed produced the highest weighted score of potential salt bridges between the apoA-I molecules (Segrest et al. 1999). This is called the LL5/5 (G129j) registry and, interestingly, is the same orientation found in the Borhani crystal structure (Borhani et al. 1997). This orientation was experimentally validated in three studies that utilised chemical cross-linking to derive the molecular orientation of the two apoA-I molecules in these particles. Two from our laboratory were highly consistent with the $5 / 5$ orientation across most of the molecule, although certain cross-links were also consistent with a second 5/2 registry (Davidson and Hilliard 2003; Silva et al. 2005). Cross-linking studies by Bhat et al. (2005) were also consistent with the general $5 / 5$ belt model for most of the molecule, but these investigators proposed that the $\mathrm{N}$ - and $\mathrm{C}$-terminal 40-50 residues doubled back on the molecule (Fig. 3b). This refinement was called the "belt and buckle". Electron paramagnetic resonance studies performed by Martin and colleagues (Martin et al. 2006) on $96 \AA$ rHDL particles were also consistent with a $5 / 5$ antiparallel double belt orientation over most of the particle circumference. However, they observed that the spin coupling signatures of residues 134-145 (within helix 5) were consistent with a looping region that causes a localised opening between the parallel belts. This "looped belt" was proposed to be a potential site by which LCAT could gain access to the cholesterol and phospholipid acyl chains that are otherwise buried in the lipid bilayer (Fig. 3c). Using hydrogen/deuterium exchange (HDX) experiments, Wu and colleagues proposed an alternative refinement of the double belt model which included the full-length apoA-I (Wu et al. 2007). This "solar flares" model again featured a $5 / 5$ double belt for most of the particle, but the N-termini were modelled as globular nodules (Fig. 3d). The HDX profile of the region between 165 and 
180 suggested that this region loops off the particle edge and could be a site for LCAT interaction. This argument was bolstered by results showing that added LCAT could alter the exchange patterns of these residues and that mutations in this region affected LCAT activity. The conclusions of this study were called into some question by a more recent HDX study performed by Chetty et al. on similar particles that failed to support the existence of the looped solar flares (Chetty et al. 2013). They showed that almost all of apoA-I was helical in the discs with the exception of the first 6 and last 7 residues. Interestingly, the region between residues 125 and 158 exhibited bimodal exchange kinetics, suggesting that this region flits between a helical and less ordered structure. This is consistent with the looped belt idea (Martin et al. 2006), but the putative solar flares region (Wu et al. 2007) was not found to be particularly dynamic in this study. The authors suggested that this region is responsible for absorbing changes in particle size by unfolding under conditions when lipid surface area is limiting. This is consistent with previous studies from our laboratory which used lipid-based fluorescence quenching agents to show that this same region changes its exposure to lipid when the particle diameter is reduced (Maiorano et al. 2004).

The recent publication of a high-resolution $(2.2 \AA)$ crystal structure of a lipidfree truncation mutant of apoA-I ( $\Delta 185-243)$ also strongly supports the double belt model (Mei and Atkinson 2011). Although lipid-free, the dimeric apoA-I formed a curved structure derived from long, kinked alpha helices. Interestingly, much of the structure resembled the $5 / 5$ double belt, even down to a similar degree of curvature. The model also suggested increased temperature factors in helix 5 hinting that there could be a separation of the helices at this site, consistent with the looped belt idea. This advance should prove to be a valuable guide for future modelling of both the lipid-free and lipid-bound forms of apoA-I.

The models discussed above conform to the generally accepted idea that these rHDLs are discoid in shape. However, Hazen and colleagues proposed a model that departed from this idea (Wu et al. 2009). Based on small-angle neutron scattering (SANS), they proposed that antiparallel apoA-I molecules form a double superhelix (DSH) that encapsulates a cigar-shaped micelle of phospholipids (Fig. 3e). Thus, the particles resemble an ellipsoid rather than a disc. Despite the different shape, the overall molecular interactions along the interface of the two apoA-I molecules are the same as the $5 / 5$ double belt model. However, unlike in the planar disc, the $\mathrm{N}$ and C-termini are not predicted interact in the DSH. The viability of this model remains under debate as molecular dynamics studies suggest that this model rapidly collapses to a disc-shaped structure (Jones et al. 2010). Even simulations done by the group that proposed the model seem to indicate that the elongated structure collapses to an oblate spheroid, though they argue it remains micellar (Gogonea et al. 2010). Going forward, this model must be squared with observations of disclike shapes by electron microscopy (Zhang et al. 2011), EPR data (Jones et al. 2010), and the quantised changes in particle size that can be readily demonstrated by reconstituting different lipid to protein ratios (Wald et al. 1990).

In addition to experimental work, significant progress has been made using molecular dynamics to simulate the structure of rHDL discs. The earliest 
simulations by Klon et al. utilised apoA-I lacking the N-terminal 43 amino acids (Klon et al. 2002b). The short $1 \mathrm{~ns}$ simulations were performed using the $5 / 5$ double belt model, encapsulating simulated phospholipids in a standard 96 ̊ particle as a starting point. The simulations provided four major conclusions. First, the overall structure remained intact over the course of the simulation, arguing the physical plausibility of the structure. Second, specific salt bridges were found to be important with regard to the molecular registry of the two apoA-I molecules. Third, the punctuating proline residues were found to induce peri-helical "kinks" that also played a role in molecular registry. Finally, the motions of the molecule suggested that apoA-I may have the potential to change its molecular registry. This concept was first proposed in the fluorescence resonance energy transfer experiments of $\mathrm{Li}$ et al. (2002), and the concept was later supported by certain chemical cross-links (Silva et al. 2005). This idea of molecular registry shifts on the HDL particle surface raises fascinating implications with respect to alternative interactions with the wide array of HDL docking proteins.

Further simulations in which lipids were added to or subtracted from the system showed that apoA-I can twist into a saddle-shaped structure in response to lipid removal (Gu et al. 2010). These results were supported by independent studies (Miyazaki et al. 2010) and in subsequent temperature jump studies using full-length apoA-I (Jones et al. 2011). Segrest and colleagues concluded that apoA-I maintains minimal surface bilayers and responds to changes in surface lipid content by winding or unwinding of this twisted saddle shape. It is important to emphasise, however, that these twisted models of dimeric apoA-I maintain similar intermolecular contacts as the planar double belt model and thus are consistent with the fluorescence, EPR and cross-linking data described above.

\subsubsection{Spherical rHDL}

Although the majority of HDL particles in human plasma are spherical, these particles are extremely understudied from a structural point of view. Spherical HDL particles contain a neutral lipid core composed of cholesteryl ester and triglyceride and thus lack a particle "edge" to constrain the apolipoproteins as in the discs. The helical domains have the potential to spread across the phospholipid surface penetrating past the phosphate group to interact with the acyl chains. Segrest et al. (1994) argued early on that if apoA-I exists in a belt-like model in discoidal particles, the fundamental interactions of apoA-I helices with the phospholipid acyl chains should not change significantly in spheres. Brasseur (Brasseur et al. 1990) and Borhani (Borhani et al. 1997) have also argued the similarity in apoA-I structure between the two shapes. These arguments were supported by circular dichroism and fluorescence studies showing that apoA-I secondary structure content and the exposure of Trp residues do not undergo gross changes when a reconstituted discoidal particle converts to a sphere in the presence of LCAT (Jonas et al. 1990). In addition, careful NMR studies suggested that the changes in conformation of apoA-I between reconstituted discs and spheres are primarily limited to the apoA-I N-terminus (Sparks et al. 1992). 


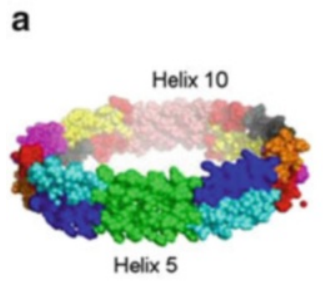

Double belt (disc)

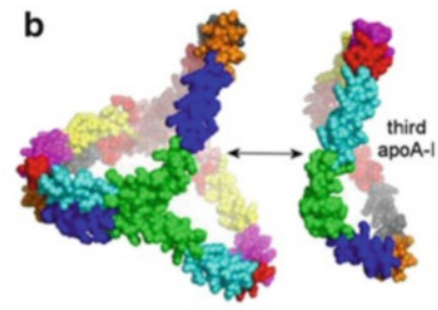

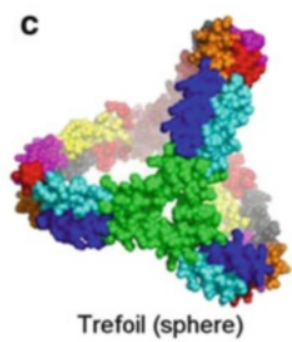

Fig. 4 The development of the trefoil model for a spherical particle from the double belt. The classic LL 5/5 double belt for apoA-I is shown in panel (a) coloured as in Fig. 3. (b) Kinks are introduced within helix 5 and helix 10 and the molecules are bent $60^{\circ}$ out of the plane of the disc. A third molecule of apoA-I, bent the same way, can now be introduced. (c) The trefoil model demonstrates similar intermolecular contacts between all three apoA-I molecules

Using the cross-linking approach, we studied the structure of reconstituted apoA-I-containing spherical particles generated by incubating rHDL discs with LCAT under controlled conditions (Silva et al. 2008). These spheres contained a largely cholesteryl ester core and were of roughly similar diameter to the "benchmark" discs described above. Cross-linking experiments showed that these spheres contained 3 molecules of apoA-I, vs. only two in the discs. Quite surprisingly, the cross-linking pattern derived in the spheres was almost identical to that of the discs. Our solution to the problem of how the exact same molecular interactions occur between two apoA-Is in a disc vs. three in a sphere was the "trefoil model" (Fig. 4). In this scheme, kinks were added in helix 5 and 10 of apoA-I. Two antiparallel belts as per the double belt model are then bent $120^{\circ}$ on these kinks and a third molecule, bent the same way, was inserted. This formed a three-dimensional cage-like structure in which the helical domains in each of the three apoA-I molecules made exactly the same salt bridge interactions postulated for the original double belt. This cage can be envisioned to encapsulate a neutral lipid ester core and support the surface polar lipids in the intervening open spaces, or lines, between the molecules.

Studying the exact same particles using SANS, $\mathrm{Wu}$ and colleagues came to a different conclusion (Wu et al. 2011). The low-resolution molecular envelope of the protein component was consistent with three different architectures: (1) two apoA-I molecules in a double belt with the third as a separate hairpin, called the helical dimer with hairpin ( $\mathrm{HdHp}$ ), (2) three separate hairpins (3Hp), or (3) an integrated trimer (iT) which is similar to the trefoil arrangement. Based on chemical crosslinking, the authors argued that the data was most consistent with the HdHp model. Despite the differences in the $\mathrm{HdHp}$ and the trefoil arrangements from a conceptual point of view, the models are quite similar from a molecular perspective in that the interactions in both models are predicted to be similar. All contacts in the trefoil are intermolecular where the interactions within the hairpined apoA-I are intramolecular, but the same sequences interact in all molecules. Thus, the epitopes on the HDL particles should be similar in both models and therefore might be expected to 
interact similarly with HDL docking proteins. It should also be noted that an alternative spherical model that is related to the trefoil organisation was proposed by Gursky (Gursky 2013) that invokes different hinge domains as extrapolated from the crystal structure of the lipid-free apoA-I fragment (Mei and Atkinson 2011). Distinction between these models awaits the development of strategies that allow for the higher resolution determination of cross-link span on particles containing more than two molecules of apoA-I.

Catte et al. made initial explorations into the structure of reconstituted spherical particles using a combination of atomistic and course-grained molecular dynamics simulations (Catte et al. 2008). The simulated particle consisted of a saddle-shaped rHDL particle with two molecules of apoA-I in which surface phospholipids were replaced in silico with cholesteryl ester (CE) molecules. During the simulations, the $\mathrm{CE}$ is sequestered into a core that was well covered by the surface protein and phospholipid, forming a prolate ellipsoid shape. The overall helicity and salt bridge orientation between the resident apoA-I molecules remained similar to the disc models, though there was an increase in overall salt bridge number. Despite an uncertain relationship with experimentally derived spherical particles, these simulations are consistent with the idea that apoA-I structures in discs and spheres are generally similar. The same group later simulated particles that more closely resembled those appearing in plasma (Segrest et al. 2013). Their data suggested that spheres created under artificial conditions in the test tube may represent kinetically trapped species with artificially high ratios of surface lipids with respect to the scaffold proteins. However, again, the resident apoA-I particles exhibited overall similar structures.

\section{3 "Real" HDL Particles}

Reconstituted HDL discs and spheres have been a critical tool for understanding the fundamental arrangements of apoA-I on the HDL particle surface. The homogeneity of these preparations allowed the use of traditional spectrophotometric approaches such as circular dichroism, NMR, EPR, etc. However, the ultimate goal has always been to understand HDL in its natural plasma form. As outlined in other sections of this chapter, human plasma HDL as isolated by density gradient ultracentrifugation is heterogeneous in almost every property: protein composition, lipid composition, particle size, particle shape and particle charge. This heterogeneity precludes the use of most traditional structural techniques. For example, circular dichroism studies of a mixed system in which apoA-I is present either in several different conformations or with other apolipoproteins will produce averaged data that will likely not be applicable to any of the particles present in the sample. Even attempts to simplify these particles, such as by immunoisolating only those particles that contain apoA-I but not apoA-II (LpA-I), still result in preparations 
with unacceptable compositional and size heterogeneity for informative structural studies. For these reasons, our understanding of HDL structure in authentic plasma HDL particles has lagged far behind the reconstituted forms.

The use of mass spectrometry as a structural tool has finally allowed this barrier to be breached. With the mass accuracy and resolution of modern mass spectrometers, it is possible to identify cross-links that are specific for a given protein, even in the presence of many others. Taking advantage of this, we studied 5 density subfractions of normal human plasma HDL as delineated by ultracentrifugation. In order to simplify the particles to the extent possible, we isolated those particles containing predominantly apoA-I (Huang et al. 2011). Surprisingly, we noted cross-linking patterns that were remarkably similar to those found in the reconstituted spheres and discs, irrespective of the particle size or density. This strongly suggested that the general features of apoA-I structure are related among lipid-associated forms of all sizes, shapes and origin. Careful measurements of the protein/lipid components indicated a range 3-5 apoA-I molecules per particle, depending on size. In fact, an important outcome of this study was the realisation that the surface of HDL particle is dominated by protein. For example, we determined that apoA-I accounts for $87 \%$ of the surface of an $\mathrm{LpA}^{\mathrm{A}} \mathrm{I}_{3 \mathrm{c}}$ particle. This only reduces to $71 \%$ in the larger $\mathrm{LpA}-\mathrm{I}_{2 \mathrm{~b}}$ particles. This is in agreement with the assertions of Segrest et al. (2013) whose compositional calculations indicate that apoA-I is much more tightly packed on native spheres than it is on typical reconstituted particles. Figure 5 shows one proposal for how 4 and 5 apoA-Is can be accommodated by changing the hinge bend angles of the trefoil while maintaining the same intermolecular interactions, and hence cross-linking pattern. Furthermore, we proposed that HDL particle size is modulated via a twisting motion of the resident apoA-Is, consistent with the MD studies of the Segrest lab (Catte et al. 2008). Combining these data, we developed the first detailed molecular models for native LpA-I HDL particles, exemplified by that for LpA-I $\mathrm{I}_{2 \mathrm{~b}}$ in Fig. 6. In this model, apoA-I forms an $11 \mathrm{~nm}$ cage stabilising 170 molecules of phospholipid at a physiological packing density. The cutaway shows a cavity of correct volume for the $\sim 143$ CEs and $\sim 23$ TGs measured in the core. These are the first detailed, molecular-scale models of native plasma HDL to be proposed.

Taking another MS approach, Chetty et al. used HDX to study spherical LpA-I particles isolated from human plasma by ultracentrifugation and further isolated by high-resolution gel filtration chromatography (Chetty et al. 2013). These particles were approximately $10 \mathrm{~nm}$ in diameter and contained on average 5 molecules of apoA-I per particle as measured by cross-linking. Comparing these directly to a benchmark rHDL disc, they found that the total helical content was similar between the native spheres and the reconstituted discs. Strikingly, the HDX profiles were also quite similar between the two with both showing near-complete helicity with the exception of the first and last 6 or so residues. Like the discs, the plasma spheres showed bimodal HDX kinetics indicating a possible loop region in helix 5 of apoA-I. Interestingly, the length of this partially disordered segment was similar 

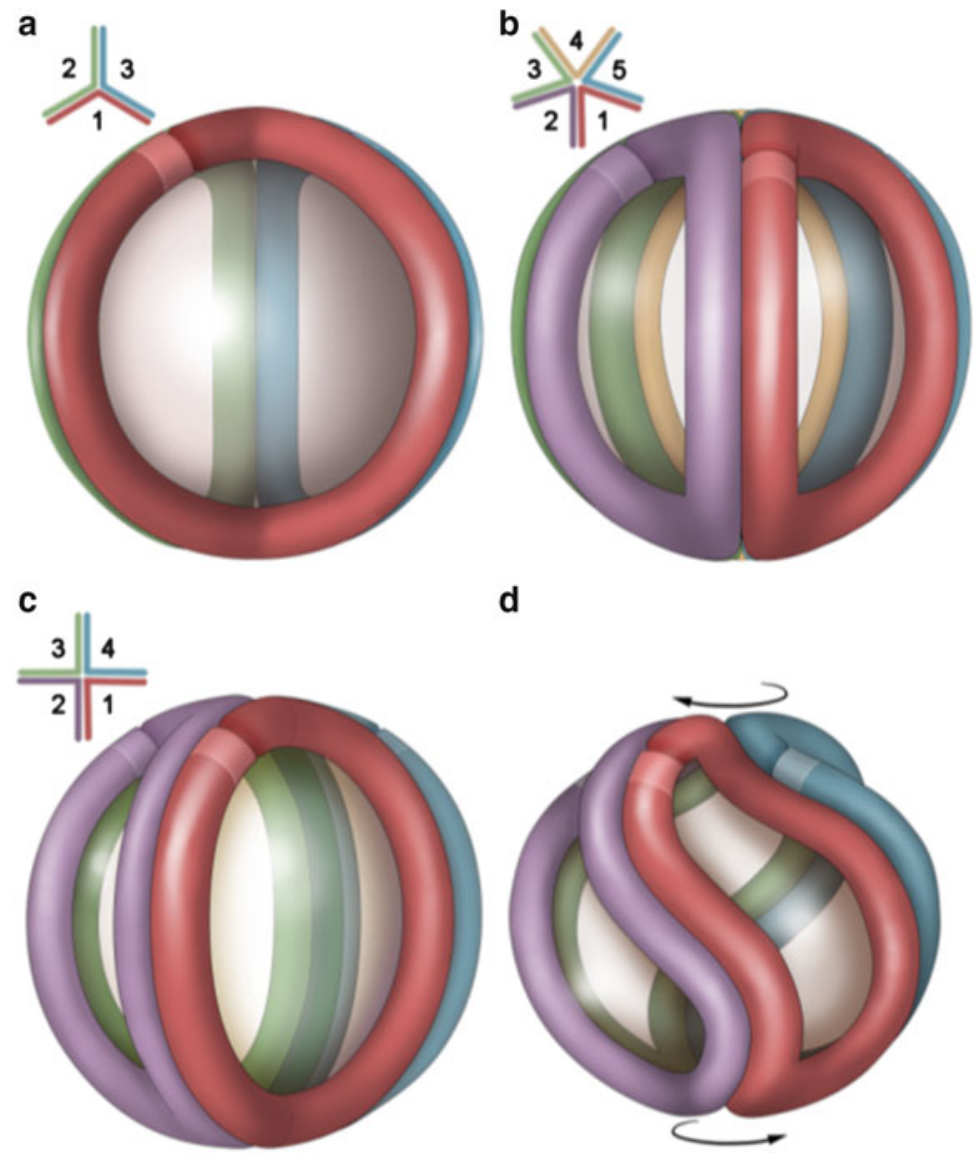

d

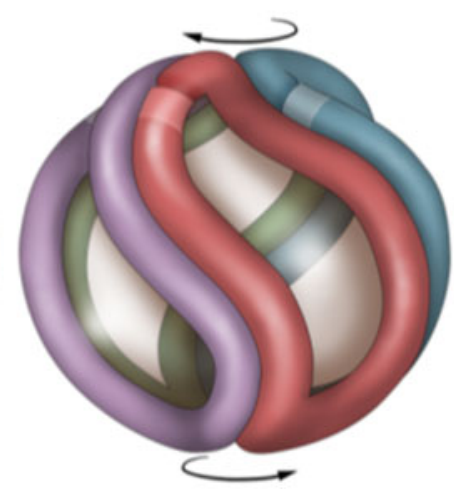

Fig. 5 Models of apoA-I in human plasma HDL particles of various size and number of apoA-I molecules. (a) The trefoil model is shown as a schematic with each molecule of apoA-I in a different colour. (b) LpA- $\mathrm{I}_{2 \mathrm{~b}}$ contains 5 apoA-I molecules, but shows a similar cross-linking patterns to reconstituted particles that have only 2 and 3 molecules of apoA-I. This figure shows that more apoA-I molecules can be added to the trefoil framework by increasing the hinge bend angle and adding more apoA-I molecules. (c) A possible model for $\mathrm{LpA}-\mathrm{I}_{2 \mathrm{a}}$ that has four apoA-I molecules on average. (d) Further reductions in HDL particle size may be accomplished by a twisting action of the resident apoA-I molecules

to that of rHDL discs of $8 \mathrm{~nm}$ in diameter, suggesting that apoA-I on these spheres is more tightly packed than in the $96 \AA$ discs. This is consistent with higher number of apoA-I molecules on the surface. Overall, these studies reinforce the idea that the global apoA-I organisation does not change significantly between particles of different shape or origin; however, more subtle conformational adaptations may occur in localised regions of the protein in response to changes in either particle diameter or surface packing density. 

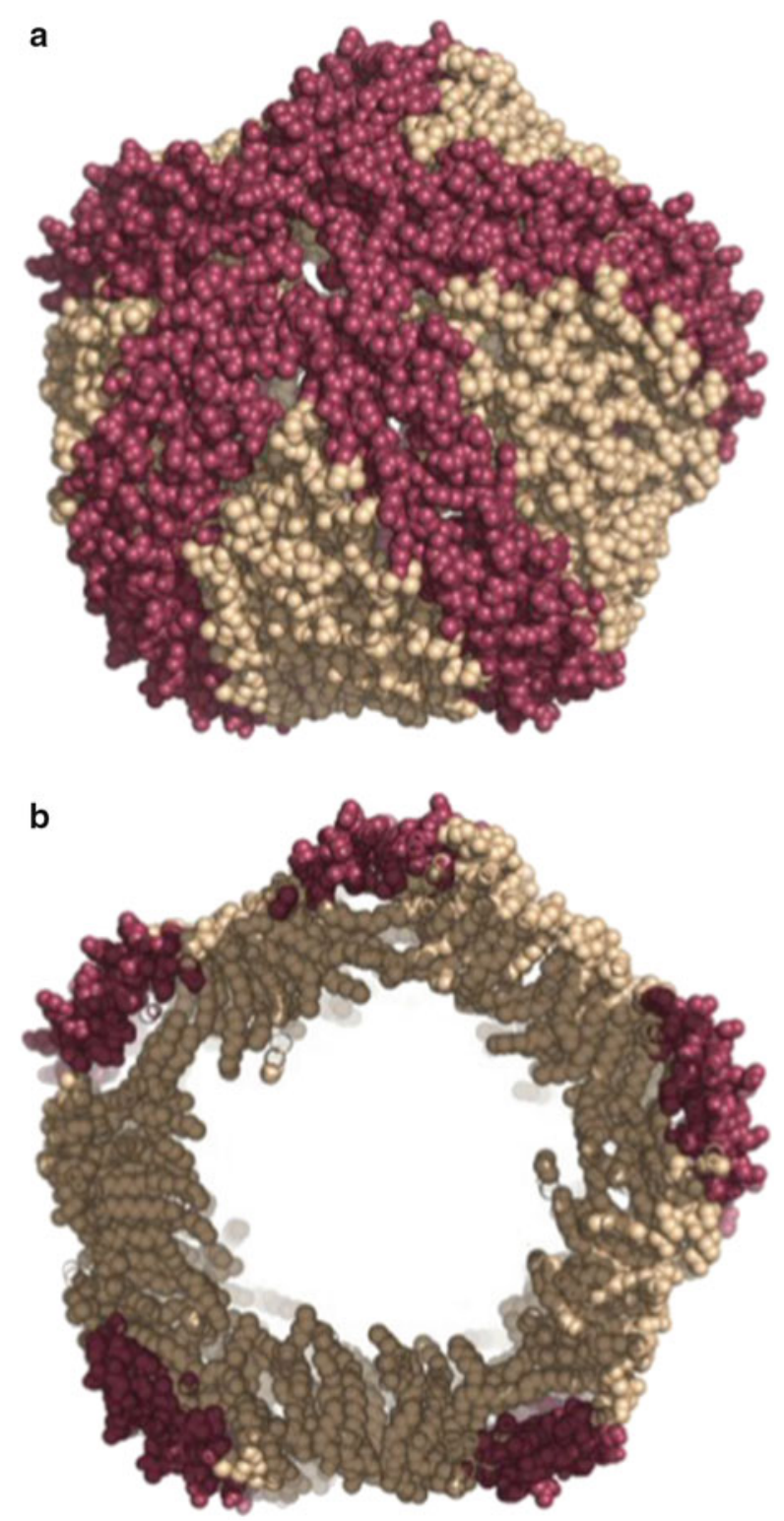

Fig. 6 Molecular model of a human plasma LpA-I $\mathrm{I}_{2 \mathrm{~b}}$ particle. (a) Five molecules of apoA-I are shown in an organisation shown schematically in Fig. 5b. Phospholipid headgroups ( $\tan )$ are present in the intervening spaces (lunes) between the apoA-I belts (purple). (b) A cross section of the same particle showing the helical belts floating atop the acyl chains of the phospholipids (brown). Note the large internal cavity that is of sufficient volume to contain the number of triglycerides and cholesteryl ester molecules that have been measured in these particles (Huang et al. 2011). (Colour figure online) 


\section{Conclusions and Perspectives}

HDL, a lipoprotein class historically and classically defined and isolated on the basis of its hydrated density alone, is a highly heterogeneous particle family consisting of multiple subclasses differing in hydrated density, size, shape and lipid and protein composition. HDL-associated proteins have been considered until now to predominate in determining the particle structure and biological function of all HDL particles. Indeed, HDL is distinguished from other lipoprotein classes by its cargo of a large number $(>80)$ of different proteins whose abundance may vary by more than 1,000 -fold and which fall into several major subgroups, including apolipoproteins, diverse enzymes, lipid transfer proteins, acute-phase response proteins, complement components and proteinase inhibitors; additional functions, such as host defence and inflammation, cannot be excluded. Many of the individual proteins are present as distinct isoforms due to co- and posttranslational modification, thereby adding a further dimension to the complex and dynamic HDL structure.

It would be erroneous however to consider that the proteome(s) of HDL particles solely determines their respective functions, particularly as cutting edge lipidomic technologies have recently revealed significant-and indeed determinant-roles of specific HDL lipid components in particle function. In this regard, negatively charged phospholipids, and notably molecular species of phosphatidylserines, are intimately associated with the cellular cholesterol efflux, antioxidative, antiapoptotic and anti-inflammatory actions of small dense HDL3 particles and equally modulate platelet activity (Camont et al. 2013). In addition, earlier studies have suggested that both core neutral lipids in HDL (cholesteryl esters and triacylglycerols) and surface lipids can impact surface fluidity and, in turn, antioxidative and other functions (ZerradSaadi et al. 2009; Camont et al. 2013).

Increasingly then, a new concept is emerging, one in which specific protein and lipid components interact closely to form individual HDL particle species, such as overall compositional and structural features translating into expression of highly defined biological functions (Camont et al. 2011; Shah et al. 2013). Clearly we are at the beginning of a new era in our understanding of HDL structure and function, with the promise of exciting mechanistic insights into function, which, in turn, will translate into new therapeutic strategies to harness the anti-atherosclerotic potential of HDL.

The central thesis that specific protein-protein interactions may drive the formation of lipoprotein complexes in the HDL particle spectrum is equally emergent. Indeed, the diversity of molecules which bind to HDL suggests that the lipoprotein can serve as a versatile adsorptive surface for proteins to form complexes playing roles not only in lipid metabolism but equally in the acutephase response, innate immune response, complement activation, proteolysis inhibition and plaque stability.

More comprehensive understanding of HDL structure is critical to future progress in HDL biology. A molecular understanding of HDL-bound apoA-I will allow further insight into its interactions with key plasma remodelling 
factors, such as LCAT, PLTP or CETP, and in addition with cell-surface proteins that mediate HDL assembly and clearance, such as ABCA1 and scavenger receptor B-I. A firm handle on these interactions would contribute to therapeutic manipulation that could enhance the protective features of HDL with respect to cardiovascular disease, or perhaps other pathologies (Gordon et al. 2011). Because HDL and its recombinant analogues have been resistant to traditional high-resolution structural approaches such as NMR or X-ray crystallography, investigators have been forced to apply a series of lower resolution techniques to the problem. As described above, these approaches have led to a general understanding of apoA-I structure in HDL that can be summarised by the following statements: (1) apoA-I pairs with itself in a double belt-like arrangement in almost all of its lipidated states; (2) apoA-I interacts via intermolecular salt bridges that commonly stabilise the registry in the $5 / 5$ orientation, though there may be a possibility of dynamic shifts to other orientations; and (3) despite a similar general organisation among different types of HDL particles, specific regions within the scaffold can undergo conformational changes depending on particle size or perhaps composition-possible sites include the $\mathrm{N}$ - and C-termini and central region in helix 5 or between residues 165 and 180. These conformationally dynamic sites are attractive possibilities for the interaction of lipid-modifying docking proteins such as LCAT and CETP.

Now that these conformationally dynamic sites within apoA-I have been proposed, it will be important to specifically define their importance for key HDL remodelling reactions. This will require well-designed site-directed mutagenesis experiments in reconstituted particles and antibody blocking experiments in native plasma particles. Furthermore, cross-linking studies hold much promise for directly mapping the sites of interaction for plasma remodelling factors on HDL-bound apoA-I.

Finally, yet another key unanswered question relates to the unknown effects of other HDL structural proteins on apoA-I structure. ApoA-II is commonly found with apoA-I on many HDL particles and would be expected to have a significant impact not only on properties such as protein packing on the particle surface but also on the conformation of apoA-I in a functionally significant way. For example, does apoA-II perform distinct structural and protein docking roles vs. apoA-I? How does each of these proteins determine the components of the bound lipidome in individual particle species? Resolution of these and other questions will continue to require the use of both reconstituted and native, authentic HDL particles combined with the appropriate application of multiple structural techniques (including continued attempts at X-ray crystallography).

It is poignant to consider that in 2014 we still lack critically important insights into HDL structure as it relates to the intravascular metabolism and atheroprotective function of these pseudomicellar, quasi-spherical particles. Had sufficient effort been invested in solving these unknowns heretofore, then perhaps our costly forays - and failures - into HDL-targeted therapeutics over the past 15 years could at least partially have been avoided. 
Acknowledgements This article results from individual contributions of all the co-authors. The highly complex world of HDL subclasses is presented by Laura Calabresi. The multifaceted composition of the HDL proteome is discussed by Anatol Kontush. Protein isoforms, including translational and posttranslational modifications, are reviewed by Mats Lindahl. Rapidly growing knowledge of the HDL lipidome is discussed by Marie Lhomme. W. Sean Davidson presents a brief history of structural studies of HDL, critically reviews existing models of HDL structure, and together with M. John Chapman draws conclusions and discusses future perspectives.

Open Access This chapter is distributed under the terms of the Creative Commons Attribution Noncommercial License, which permits any noncommercial use, distribution, and reproduction in any medium, provided the original author(s) and source are credited.

\section{References}

Ahnstrom J et al (2007) Hydrophobic ligand binding properties of the human lipocalin apolipoprotein M. J Lipid Res 48(8):1754-1762

Allan CM, Taylor JM (1996) Expression of a novel human apolipoprotein (apoC-IV) causes hypertriglyceridemia in transgenic mice. J Lipid Res 37(7):1510-1518

Argraves KM et al (2011) S1P, dihydro-S1P and C24:1-ceramide levels in the HDL-containing fraction of serum inversely correlate with occurrence of ischemic heart disease. Lipids Health Dis 10:70

Asztalos BF, Schaefer EJ (2003a) High-density lipoprotein subpopulations in pathologic conditions. Am J Cardiol 91(7A):12E-17E

Asztalos BF, Schaefer EJ (2003b) HDL in atherosclerosis: actor or bystander? Atheroscler Suppl 4 (1):21-29

Asztalos BF et al (2007) Role of LCAT in HDL remodeling: investigation of LCAT deficiency states. J Lipid Res 48(3):592-599

Atkinson D et al (1974) The structure of a high density lipoprotein (HDL3) from porcine plasma. Proc R Soc Lond B Biol Sci 186(083):165-180

Axler O et al (2007) An ELISA for apolipoprotein M reveals a strong correlation to total cholesterol in human plasma. J Lipid Res 48(8):1772-1780

Axler $\mathrm{O}$ et al (2008) Apolipoprotein M associates to lipoproteins through its retained signal peptide. FEBS Lett 582(5):826-828

Baenziger JU (2012) Moving the O-glycoproteome from form to function. Proc Natl Acad Sci USA 109(25):9672-9673

Benditt EP, Eriksen N (1977) Amyloid protein SAA is associated with high density lipoprotein from human serum. Proc Natl Acad Sci USA 74(9):4025-4028

Bergmeier C et al (2004) Distribution spectrum of paraoxonase activity in HDL fractions. Clin Chem 50(12):2309-2315

Bhat $\mathrm{S}$ et al (2005) Intermolecular contact between globular N-terminal fold and C-terminal domain of ApoA-I stabilizes its lipid-bound conformation: studies employing chemical cross-linking and mass spectrometry. J Biol Chem 280(38):33015-33025

Bisgaier CL et al (1985) Distribution of apolipoprotein A-IV in human plasma. J Lipid Res 26 (1):11-25

Bondarenko PV et al (1999) Mass spectral study of polymorphism of the apolipoproteins of very low density lipoprotein. J Lipid Res 40(3):543-555

Borhani DW et al (1997) Crystal structure of truncated human apolipoprotein A-I suggests a lipidbound conformation. Proc Natl Acad Sci USA 94(23):12291-12296

Brasseur R et al (1990) Mode of assembly of amphipathic helical segments in model high-density lipoproteins. Biochim Biophys Acta 1043(3):245-252 
Brewer HB Jr et al (1972) Amino acid sequence of human apoLp-Gln-II (apoA-II), an apolipoprotein isolated from the high-density lipoprotein complex. Proc Natl Acad Sci USA 69 (5):1304-1308

Brewer HB Jr et al (1978) The amino acid sequence of human APOA-I, an apolipoprotein isolated from high density lipoproteins. Biochem Biophys Res Commun 80(3):623-630

Brouillette CG, Anantharamaiah GM (1995) Structural models of human apolipoprotein A-I. Biochim Biophys Acta 1256(2):103-129

Brouillette CG et al (2001) Structural models of human apolipoprotein A-I: a critical analysis and review. Biochim Biophys Acta 1531(1-2):4-46

Bruneel A et al (2008) Two dimensional gel electrophoresis of apolipoprotein C-III and MALDITOF MS are complementary techniques for the study of combined defects in $\mathrm{N}$ - and mucin type O-glycan biosynthesis. Proteomics Clin Appl 2:1670-1674

Cabana VG et al (1996) HDL content and composition in acute phase response in three species: triglyceride enrichment of HDL a factor in its decrease. J Lipid Res 37(12):2662-2674

Cabana VG et al (2004) Influence of apoA-I and apoE on the formation of serum amyloid A-containing lipoproteins in vivo and in vitro. J Lipid Res 45(2):317-325

Calabresi L et al (2001) Limited proteolysis of a disulfide-linked apoA-I dimer in reconstituted HDL. J Lipid Res 42(6):935-942

Camont L et al (2011) Biological activities of HDL subpopulations and their relevance to cardiovascular disease. Trends Mol Med 17(10):594-603

Camont L et al (2013) Small, dense high-density lipoprotein-3 particles are enriched in negatively charged phospholipids: relevance to cellular cholesterol efflux, antioxidative, antithrombotic, anti-inflammatory, and antiapoptotic functionalities. Arterioscler Thromb Vasc Biol 33 (12):2715-2723

Campos E, McConathy WJ (1986) Distribution of lipids and apolipoproteins in human plasma by vertical spin ultracentrifugation. Arch Biochem Biophys 249(2):455-463

Candiano $\mathrm{G}$ et al (2008) High-resolution 2-DE for resolving proteins, protein adducts and complexes in plasma. Electrophoresis 29(3):682-694

Catte A et al (2008) Structure of spheroidal HDL particles revealed by combined atomistic and coarse-grained simulations. Biophys J 94(6):2306-2319

Chapman MJ et al (1981) A density gradient ultracentrifugal procedure for the isolation of the major lipoprotein classes from human serum. J Lipid Res 22(2):339-358

Chen N et al (2000) Physiologic concentrations of homocysteine inhibit the human plasma GSH peroxidase that reduces organic hydroperoxides. J Lab Clin Med 136(1):58-65

Chetty PS et al (2013) Comparison of apoA-I helical structure and stability in discoidal and spherical HDL particles by HX and mass spectrometry. J Lipid Res 54(6):1589-1597

Cheung MC, Albers JJ (1982) Distribution of high density lipoprotein particles with different apoprotein composition: particles with A-I and A-II and particles with A-I but no A-II. J Lipid Res 23(5):747-753

Cheung MC et al (2010) Phospholipid transfer protein in human plasma associates with proteins linked to immunity and inflammation. Biochemistry 49(34):7314-7322

Chiba $\mathrm{T}$ et al (2011) Serum amyloid A facilitates the binding of high-density lipoprotein from mice injected with lipopolysaccharide to vascular proteoglycans. Arterioscler Thromb Vasc Biol 31 (6):1326-1332

Christoffersen C et al (2008) The signal peptide anchors apolipoprotein $\mathrm{M}$ in plasma lipoproteins and prevents rapid clearance of apolipoprotein M from plasma. J Biol Chem 283(27):1876518772

Christoffersen C et al (2011) Endothelium-protective sphingosine-1-phosphate provided by HDL-associated apolipoprotein M. Proc Natl Acad Sci USA 108(23):9613-9618

Coetzee GA et al (1986) Serum amyloid A-containing human high density lipoprotein 3. Density, size, and apolipoprotein composition. J Biol Chem 261(21):9644-9651 
Collins LA et al (2010) Integrated approach for the comprehensive characterization of lipoproteins from human plasma using FPLC and nano-HPLC-tandem mass spectrometry. Physiol Genomics 40(3):208-215

Contiero E et al (1997) Apolipoprotein AI isoforms in serum determined by isoelectric focusing and immunoblotting. Electrophoresis 18(1):122-126

Dahlback B, Nielsen LB (2009) Apolipoprotein M affecting lipid metabolism or just catching a ride with lipoproteins in the circulation? Cell Mol Life Sci 66(4):559-564

Davidson WS, Hilliard GM (2003) The spatial organization of apolipoprotein A-I on the edge of discoidal high density lipoprotein particles: a mass specrometry study. J Biol Chem 278 (29):27199-27207

Davidson WS et al (1995) Effects of acceptor particle size on the efflux of cellular free cholesterol. J Biol Chem 270(29):17106-17113

Davidson WS et al (2009) Proteomic analysis of defined HDL subpopulations reveals particlespecific protein clusters: relevance to antioxidative function. Arterioscler Thromb Vasc Biol 29 (6):870-876

Davidsson P et al (2010) Proteomics of apolipoproteins and associated proteins from plasma highdensity lipoproteins. Arterioscler Thromb Vasc Biol 30(2):156-163

de Beer MC et al (2010) Impact of serum amyloid A on high density lipoprotein composition and levels. J Lipid Res 51(11):3117-3125

De Lalla OF, Gofman JW (1954) Ultracentrifugal analysis of serum lipoproteins. Methods Biochem Anal 1:459-478

de Seny D et al (2008) Monomeric calgranulins measured by SELDI-TOF mass spectrometry and calprotectin measured by ELISA as biomarkers in arthritis. Clin Chem 54(6):1066-1075

de Silva HV et al (1990a) Purification and characterization of apolipoprotein J. J Biol Chem 265 (24):14292-14297

de Silva HV et al (1990b) A 70-kDa apolipoprotein designated ApoJ is a marker for subclasses of human plasma high density lipoproteins. J Biol Chem 265(22):13240-13247

Deguchi $\mathrm{H}$ et al (2000) Cardiolipin is a normal component of human plasma lipoproteins. Proc Natl Acad Sci USA 97(4):1743-1748

Duchateau PN et al (1997) Apolipoprotein L, a new human high density lipoprotein apolipoprotein expressed by the pancreas. Identification, cloning, characterization, and plasma distribution of apolipoprotein L. J Biol Chem 272(41):25576-25582

Ducret A et al (1996) Characterization of human serum amyloid A protein isoforms separated by two-dimensional electrophoresis by liquid chromatography/electrospray ionization tandem mass spectrometry. Electrophoresis 17(5):866-876

Duriez P, Fruchart JC (1999) High-density lipoprotein subclasses and apolipoprotein A-I. Clin Chim Acta 286(1-2):97-114

Edelstein C et al (1972) On the subunit structure of the protein of human serum high density lipoprotein. I. A study of its major polypeptide component (Sephadex, fraction 3). J Biol Chem 247(18):5842-5849

Favari E et al (2004) Depletion of pre-beta-high density lipoprotein by human chymase impairs ATP-binding cassette transporter A1- but not scavenger receptor class B type I-mediated lipid efflux to high density lipoprotein. J Biol Chem 279(11):9930-9936

Favari E et al (2007) A unique protease-sensitive high density lipoprotein particle containing the apolipoprotein A-I Milano dimer effectively promotes ATP-binding Cassette A1-mediated cell cholesterol efflux. J Biol Chem 282(8):5125-5132

Fernandez-Irigoyen J et al (2005) Oxidation of specific methionine and tryptophan residues of apolipoprotein A-I in hepatocarcinogenesis. Proteomics 5(18):4964-4972

Fievet C et al (1995) Non-enzymatic glycosylation of apolipoprotein A-I and its functional consequences. Diabete Metab 21(2):95-98

Flood-Nichols SK et al (2013) Longitudinal analysis of maternal plasma apolipoproteins in pregnancy: a targeted proteomics approach. Mol Cell Proteomics 12(1):55-64 
Forte TM, Nordhausen RW (1986) Electron microscopy of negatively stained lipoproteins. Methods Enzymol 128:442-457

Franceschini G et al (1985) Effects of storage on the distribution of high density lipoprotein subfractions in human sera. J Lipid Res 26(11):1368-1373

Franceschini $\mathrm{G}$ et al (2007) Effects of fenofibrate and simvastatin on HDL-related biomarkers in low-HDL patients. Atherosclerosis 195(2):385-391

Gauthamadasa K et al (2010) Speciated human high-density lipoprotein protein proximity profiles. Biochemistry 49(50):10656-10665

Ghiselli G et al (1985) Origin of apolipoprotein A-I polymorphism in plasma. J Biol Chem 260 (29): $15662-15668$

Gidez LI et al (1982) Separation and quantitation of subclasses of human plasma high density lipoproteins by a simple precipitation procedure. J Lipid Res 23(8):1206-1223

Gillard BK et al (2005) Plasma factors required for human apolipoprotein A-II dimerization. Biochemistry 44(2):471-479

Gogonea V et al (2010) Congruency between biophysical data from multiple platforms and molecular dynamics simulation of the double-super helix model of nascent high-density lipoprotein. Biochemistry 49(34):7323-7343

Gordon JI et al (1983) Biosynthesis of human preproapolipoprotein A-II. J Biol Chem 258 (22):14054-14059

Gordon SM et al (2010) Proteomic characterization of human plasma high density lipoprotein fractionated by gel filtration chromatography. J Proteome Res 9(10):5239-5249

Gordon SM et al (2011) High density lipoprotein: it's not just about lipid transport anymore. Trends Endocrinol Metab 22(1):9-15

Gordon SM et al (2013) Multi-dimensional co-separation analysis reveals protein-protein interactions defining plasma lipoprotein subspecies. Mol Cell Proteomics 12(11):3123-3134

Goswami B et al (2009) Paraoxonase: a multifaceted biomolecule. Clin Chim Acta 410(1-2):1-12

Gu F et al (2010) Structures of discoidal high density lipoproteins: a combined computationalexperimental approach. J Biol Chem 285(7):4652-4665

Gursky O (2013) Crystal structure of Delta(185-243)ApoA-I suggests a mechanistic framework for the protein adaptation to the changing lipid load in good cholesterol: from flatland to sphereland via double belt, belt buckle, double hairpin and trefoil/tetrafoil. J Mol Biol 425 (1): $1-16$

Gursky O et al (2013) Structural basis for distinct functions of the naturally occurring Cys mutants of human apolipoprotein A-I. J Lipid Res 54(12):3244-3257

Hajduk SL et al (1989) Lysis of Trypanosoma brucei by a toxic subspecies of human high density lipoprotein. J Biol Chem 264(9):5210-5217

Halim A et al (2013) LC-MS/MS characterization of O-glycosylation sites and glycan structures of human cerebrospinal fluid glycoproteins. J Proteome Res 12(2):573-584

Harel M et al (2004) Structure and evolution of the serum paraoxonase family of detoxifying and anti-atherosclerotic enzymes. Nat Struct Mol Biol 11(5):412-419

Harvey SB et al (2009) O-glycoside biomarker of apolipoprotein C3: responsiveness to obesity, bariatric surgery, and therapy with metformin, to chronic or severe liver disease and to mortality in severe sepsis and graft vs host disease. J Proteome Res 8(2):603-612

Hatters DM et al (2006) Apolipoprotein E structure: insights into function. Trends Biochem Sci 31 (8):445-454

He Y et al (2008) Control of cholesteryl ester transfer protein activity by sequestration of lipid transfer inhibitor protein in an inactive complex. J Lipid Res 49(7):1529-1537

Heinecke JW (2009) The HDL proteome: a marker - and perhaps mediator - of coronary artery disease. J Lipid Res Supp 50:S167-S171

Hennessy LK et al (1997) Isolation of subpopulations of high density lipoproteins: three particle species containing apoE and two species devoid of apoE that have affinity for heparin. J Lipid Res 38(9):1859-1868 
Hoffman JS, Benditt EP (1982) Changes in high density lipoprotein content following endotoxin administration in the mouse. Formation of serum amyloid protein-rich subfractions. J Biol Chem 257(17):10510-10517

Holleboom AG et al (2011) Heterozygosity for a loss-of-function mutation in GALNT2 improves plasma triglyceride clearance in man. Cell Metab 14(6):811-818

Holmquist L (2002) Selective extraction of lecithin:cholesterol acyltransferase (EC 2.3.1.43) from human plasma. J Biochem Biophys Methods 52(1):63-68

Hoofnagle AN, Heinecke JW (2009) Lipoproteomics: using mass spectrometry-based proteomics to explore the assembly, structure, and function of lipoproteins. J Lipid Res 50(10):1967-1975

Hortin GL et al (2006) Diverse range of small peptides associated with high-density lipoprotein. Biochem Biophys Res Commun 340(3):909-915

Huang R et al (2011) Apolipoprotein A-I structural organization in high-density lipoproteins isolated from human plasma. Nat Struct Mol Biol 18(4):416-422

Hussain MM, Zannis VI (1990) Intracellular modification of human apolipoprotein AII (apoAII) and sites of apoAII mRNA synthesis: comparison of apoAII with apoCII and apoCIII isoproteins. Biochemistry 29(1):209-217

Jakubowski H (2000) Calcium-dependent human serum homocysteine thiolactone hydrolase. A protective mechanism against protein N-homocysteinylation. J Biol Chem 275(6):3957-3962

Jaleel A et al (2010) Identification of de novo synthesized and relatively older proteins: accelerated oxidative damage to de novo synthesized apolipoprotein A-1 in type 1 diabetes. Diabetes 59 (10):2366-2374

James RW et al (1988) Protein heterogeneity of lipoprotein particles containing apolipoprotein A-I without apolipoprotein A-II and apolipoprotein A-I with apolipoprotein A-II isolated from human plasma. J Lipid Res 29(12):1557-1571

Jian W et al (2013) Relative quantitation of glycoisoforms of intact apolipoprotein C3 in human plasma by liquid chromatography-high-resolution mass spectrometry. Anal Chem 85(5):28672874

Jin Y, Manabe T (2005) Direct targeting of human plasma for matrix-assisted laser desorption/ ionization and analysis of plasma proteins by time of flight-mass spectrometry. Electrophoresis 26(14):2823-2834

Jonas A (1972) Studies on the structure of bovine serum high density lipoprotein using covalently bound fluorescent probes. J Biol Chem 247(23):7773-7778

Jonas A (1986) Reconstitution of high-density lipoproteins. Methods Enzymol 128:553-582

Jonas A (2002) Lipoprotein structure. In: Vance DE, Vance JE (eds) Biochemistry of lipids, lipoproteins and membranes. Elsevier, Amsterdam, pp 483-504

Jonas A, McHugh HT (1984) Reaction of lecithin: cholesterol acyltransferase with micellar substrates. Effect of particle sizes. Biochim Biophys Acta 794(3):361-372

Jonas A et al (1989) Defined apolipoprotein A-I conformations in reconstituted high density lipoprotein discs. J Biol Chem 264(9):4818-4824

Jonas A et al (1990) Apolipoprotein A-I structure and lipid properties in homogeneous, reconstituted spherical and discoidal high density lipoproteins. J Biol Chem 265(36):2212322129

Jones MK et al (2010) Assessment of the validity of the double superhelix model for reconstituted high density lipoproteins: a combined computational-experimental approach. J Biol Chem 285 (52):41161-41171

Jones MK et al (2011) "Sticky" and "promiscuous", the Yin and Yang of apolipoprotein A-I termini in discoidal high-density lipoproteins: a combined computational-experimental approach. Biochemistry 50(12):2249-2263

Karlsson $\mathrm{H}$ et al (2005) Lipoproteomics II: mapping of proteins in high-density lipoprotein using two-dimensional gel electrophoresis and mass spectrometry. Proteomics 5(5):1431-1445

Karlsson $\mathrm{H}$ et al (2009) Protein profiling of low-density lipoprotein from obese subjects. Proteomics Clin Appl 3(6):663-671 
Khovidhunkit W et al (2004) Effects of infection and inflammation on lipid and lipoprotein metabolism: mechanisms and consequences to the host. J Lipid Res 45(7):1169-1196

Kiernan UA et al (2003) Detection of novel truncated forms of human serum amyloid A protein in human plasma. FEBS Lett 537(1-3):166-170

$\mathrm{Kim} \mathrm{E}$ et al (2008) Expression of apolipoprotein C-IV is regulated by $\mathrm{Ku}$ antigen/peroxisome proliferator-activated receptor gamma complex and correlates with liver steatosis. J Hepatol 49 (5):787-798

Kisilevsky R, Manley PN (2012) Acute-phase serum amyloid A: perspectives on its physiological and pathological roles. Amyloid 19(1):5-14

Kisilevsky R, Tam SP (2003) Macrophage cholesterol efflux and the active domains of serum amyloid A 2.1. J Lipid Res 44(12):2257-2269

Klon AE et al (2002a) Comparative models for human apolipoprotein A-I bound to lipid in discoidal high-density lipoprotein particles. Biochemistry 41(36):10895-10905

Klon AE et al (2002b) Molecular dynamics simulations on discoidal HDL particles suggest a mechanism for rotation in the apo A-I belt model. J Mol Biol 324(4):703-721

Kontush A, Chapman MJ (2010) Antiatherogenic function of HDL particle subpopulations: focus on antioxidative activities. Curr Opin Lipidol 21(4):312-318

Kontush A, Chapman MJ (2012) High-density lipoproteins: structure, metabolism, function and therapeutics. Wiley, New York

Kontush A et al (2003) Small, dense HDL particles exert potent protection of atherogenic LDL against oxidative stress. Arterioscler Thromb Vasc Biol 23(10):1881-1888

Kontush A et al (2007) Preferential sphingosine-1-phosphate enrichment and sphingomyelin depletion are key features of small dense HDL3 particles: relevance to antiapoptotic and antioxidative activities. Arterioscler Thromb Vasc Biol 27(8):1843-1849

Koppaka V et al (1999) The structure of human lipoprotein A-I. Evidence for the "belt" model. J Biol Chem 274(21):14541-14544

Kulkarni KR et al (1997) Quantification of HDL2 and HDL3 cholesterol by the vertical auto profile-II (VAP-II) methodology. J Lipid Res 38(11):2353-2364

Laggner P et al (1973) Studies on the structure of lipoprotein A of human high density lipoprotein HDL3: the spherically averaged electron density distribution. FEBS Lett 33(1):77-80

Lagor WR et al (2009) Overexpression of apolipoprotein F reduces HDL cholesterol levels in vivo. Arterioscler Thromb Vasc Biol 29(1):40-46

Lamant $\mathrm{M}$ et al (2006) ApoO, a novel apolipoprotein, is an original glycoprotein up-regulated by diabetes in human heart. J Biol Chem 281(47):36289-36302

Lee $\mathrm{M}$ et al (2003) Apolipoprotein composition and particle size affect HDL degradation by chymase: effect on cellular cholesterol efflux. J Lipid Res 44(3):539-546

Lee JY et al (2010) Profiling of phospholipids in lipoproteins by multiplexed hollow fiber flow field-flow fractionation and nanoflow liquid chromatography-tandem mass spectrometry. J Chromatogr A 1217(10):1660-1666

Levels JH et al (2011) High-density lipoprotein proteome dynamics in human endotoxemia. Proteome Sci 9(1):34

Lhomme $\mathrm{M}$ et al (2012) Lipidomics in lipoprotein biology. In: Ekroos K (ed) Lipidomics: technologies and applications. Wiley, Weinheim, pp 197-231

$\mathrm{Li} \mathrm{H}$ et al (2000) Structural determination of lipid-bound ApoA-I using fluorescence resonance energy transfer. J Biol Chem 275(47):37048-37054

Li HH et al (2002) ApoA-I structure on discs and spheres. Variable helix registry and conformational states. J Biol Chem 277(42):39093-39101

Liadaki KN et al (2000) Binding of high density lipoprotein (HDL) and discoidal reconstituted HDL to the HDL receptor scavenger receptor class B type I. Effect of lipid association and APOA-I mutations on receptor binding. J Biol Chem 275(28):21262-21271

Lipina C, Hundal HS (2011) Sphingolipids: agents provocateurs in the pathogenesis of insulin resistance. Diabetologia 54(7):1596-1607 
Ljunggren S et al (2013) APOA-I mutations, L202P and K131DEL, IN HDL from heterozygotes with low HDL-cholesterol. Proteomics Clin Appl 8(3-4):241-250

Lucke S, Levkau B (2010) Endothelial functions of sphingosine-1-phosphate. Cell Physiol Biochem 26(1):87-96

Lund-Katz S, Phillips MC (2010) High density lipoprotein structure-function and role in reverse cholesterol transport. Subcell Biochem 51:183-227

Mackness B et al (2010) Human tissue distribution of paraoxonases 1 and 2 mRNA. IUBMB Life 62(6):480-482

Maeba R, Ueta N (2003) Ethanolamine plasmalogen and cholesterol reduce the total membrane oxidizability measured by the oxygen uptake method. Biochem Biophys Res Commun 302 (2):265-270

Maiorano JN et al (2004) Identification and structural ramifications of a hinge domain in apolipoprotein A-I discoidal high-density lipoproteins of different size. Biochemistry 43(37):1171711726

Majek P et al (2011) Plasma proteome changes in cardiovascular disease patients: novel isoforms of apolipoprotein A1. J Transl Med 9:84

Mallat Z et al (2010) Lipoprotein-associated and secreted phospholipases A in cardiovascular disease: roles as biological effectors and biomarkers. Circulation 122(21):2183-2200

Marcel YL et al (1990) Distribution and concentration of cholesteryl ester transfer protein in plasma of normolipemic subjects. J Clin Invest 85(1):10-17

Marhaug $\mathrm{G}$ et al (1982) Characterization of amyloid related protein SAA complexed with serum lipoproteins (apoSAA). Clin Exp Immunol 50(2):382-389

Martin DD et al (2006) Apolipoprotein A-I assumes a "looped belt" conformation on reconstituted high density lipoprotein. J Biol Chem 281(29):20418-20426

Matz CE, Jonas A (1982) Micellar complexes of human apolipoprotein A-I with phosphatidylcholines and cholesterol prepared from cholate-lipid dispersions. J Biol Chem 257(8):45354540

Mauger JF et al (2006) Apolipoprotein C-III isoforms: kinetics and relative implication in lipid metabolism. J Lipid Res 47(6):1212-1218

Mazur MT et al (2010) Quantitative analysis of intact apolipoproteins in human HDL by top-down differential mass spectrometry. Proc Natl Acad Sci USA 107(17):7728-7733

McConathy WJ, Alaupovic P (1973) Isolation and partial characterization of apolipoprotein D: a new protein moiety of the human plasma lipoprotein system. FEBS Lett 37(2):178-182

McIntyre TM et al (2009) The emerging roles of PAF acetylhydrolase. J Lipid Res Supp 50:S255S259

McLachlan AD (1977) Repeated helical pattern in apolipoprotein-A-I. Nature 267(5610):465-466

Mei X, Atkinson D (2011) Crystal structure of C-terminal truncated apolipoprotein A-I reveals the assembly of high density lipoprotein (HDL) by dimerization. J Biol Chem 286(44):38570 38582

Miida T et al (2003) Analytical performance of a sandwich enzyme immunoassay for pre beta 1-HDL in stabilized plasma. J Lipid Res 44(3):645-650

Miyazaki M et al (2010) Static and dynamic characterization of nanodiscs with apolipoprotein A-I and its model peptide. J Phys Chem B 114(38):12376-12382

Mora S (2009) Advanced lipoprotein testing and subfractionation are not (yet) ready for routine clinical use. Circulation 119(17):2396-2404

Moren X et al (2008) HDL subfraction distribution of paraoxonase-1 and its relevance to enzyme activity and resistance to oxidative stress. J Lipid Res 49(6):1246-1253

Navab M et al (2004) The oxidation hypothesis of atherogenesis: the role of oxidized phospholipids and HDL. J Lipid Res 45(6):993-1007

Nelsestuen GL et al (2008) Top-down proteomic analysis by MALDI-TOF profiling: concentration-independent biomarkers. Proteomics Clin Appl 2(2):158-166

Neumann S et al (2009) Mammalian Wnt3a is released on lipoprotein particles. Traffic 10(3):334343 
Nicholls SJ, Hazen SL (2009) Myeloperoxidase, modified lipoproteins, and atherogenesis. J Lipid Res Supp 50:S346-S351

Nichols AV et al (1983) Characterization of discoidal complexes of phosphatidylcholine, apolipoprotein A-I and cholesterol by gradient gel electrophoresis. Biochim Biophys Acta 750 (2):353-364

Nichols AV et al (1986) Nondenaturing polyacrylamide gradient gel electrophoresis. Methods Enzymol 128:417-431

Nicolardi S et al (2013a) Mapping O-glycosylation of apolipoprotein C-III in MALDI-FT-ICR protein profiles. Proteomics 13(6):992-1001

Nicolardi S et al (2013b) Identification of new apolipoprotein-CIII glycoforms with ultrahigh resolution MALDI-FTICR mass spectrometry of human sera. J Proteome Res 12(5):22602268

Nijstad N et al (2011) Overexpression of apolipoprotein O does not impact on plasma HDL levels or functionality in human apolipoprotein A-I transgenic mice. Biochim Biophys Acta 1811 (4):294-299

Nilsson A, Duan RD (2006) Absorption and lipoprotein transport of sphingomyelin. J Lipid Res 47 (1):154-171

Nobecourt E et al (2007) The impact of glycation on apolipoprotein A-I structure and its ability to activate lecithin:cholesterol acyltransferase. Diabetologia 50(3):643-653

Nolte RT, Atkinson D (1992) Conformational analysis of apolipoprotein A-I and E-3 based on primary sequence and circular dichroism. Biophys J 63(5):1221-1239

Ohta T et al (1985) Studies on the in vivo and in vitro distribution of apolipoprotein A-IV in human plasma and lymph. J Clin Invest 76(3):1252-1260

Olofsson SO et al (1978) Isolation and partial characterization of a new acidic apolipoprotein (apolipoprotein F) from high density lipoproteins of human plasma. Biochemistry 17(6):10321036

Ortiz-Munoz G et al (2009) HDL antielastase activity prevents smooth muscle cell anoikis, a potential new antiatherogenic property. FASEB J 23(9):3129-3139

Otvos JD et al (1992) Development of a proton nuclear magnetic resonance spectroscopic method for determining plasma lipoprotein concentrations and subspecies distributions from a single, rapid measurement. Clin Chem 38(9):1632-1638

Panagotopulos SE et al (2001) Apolipoprotein A-I adopts a belt-like orientation in reconstituted high density lipoproteins. J Biol Chem 276(46):42965-42970

Park KH et al (2010) Fructated apolipoprotein A-I showed severe structural modification and loss of beneficial functions in lipid-free and lipid-bound state with acceleration of atherosclerosis and senescence. Biochem Biophys Res Commun 392(3):295-300

Phillips MC (2013) New insights into the determination of HDL structure by apolipoproteins: thematic review series: high density lipoprotein structure, function, and metabolism. J Lipid Res 54(8):2034-2048

Phillips JC et al (1997) Predicting the structure of apolipoprotein A-I in reconstituted high-density lipoprotein disks. Biophys J 73(5):2337-2346

Pruzanski W et al (2000) Comparative analysis of lipid composition of normal and acute-phase high density lipoproteins. J Lipid Res 41(7):1035-1047

Puppione DL et al (2010) Mass spectral analyses of the two major apolipoproteins of great ape high density lipoproteins. Comp Biochem Physiol Part D Genomics Proteomics 4(4):305-309

Qiu X et al (2007) Crystal structure of cholesteryl ester transfer protein reveals a long tunnel and four bound lipid molecules. Nat Struct Mol Biol 14(2):106-113

Rassart E et al (2000) Apolipoprotein D. Biochim Biophys Acta 1482(1-2):185-198

Remaley AT et al (1993) O-linked glycosylation modifies the association of apolipoprotein A-II to high density lipoproteins. J Biol Chem 268(9):6785-6790

Roghani A, Zannis VI (1988) Mutagenesis of the glycosylation site of human ApoCIII. O-linked glycosylation is not required for ApoCIII secretion and lipid binding. J Biol Chem 263 (34):17925-17932 
Rosenson RS et al (2011) HDL measures, particle heterogeneity, proposed nomenclature, and relation to atherosclerotic cardiovascular events. Clin Chem 57(3):392-410

Rousset X et al (2009) Lecithin: cholesterol acyltransferase-from biochemistry to role in cardiovascular disease. Curr Opin Endocrinol Diabetes Obes 16(2):163-171

Rye KA et al (1996) The influence of sphingomyelin on the structure and function of reconstituted high density lipoproteins. J Biol Chem 271(8):4243-4250

Saito $\mathrm{H}$ et al (2000) Inhibition of lipoprotein lipase activity by sphingomyelin: role of membrane surface structure. Biochim Biophys Acta 1486(2-3):312-320

Samanta U, Bahnson BJ (2008) Crystal structure of human plasma platelet-activating factor acetylhydrolase: structural implication to lipoprotein binding and catalysis. J Biol Chem 283 (46):31617-31624

Santucci L et al (2011) Protein-protein interaction heterogeneity of plasma apolipoprotein A1 in nephrotic syndrome. Mol Biosyst 7(3):659-666

Sattler KJ et al (2010) Sphingosine 1-phosphate levels in plasma and HDL are altered in coronary artery disease. Basic Res Cardiol 105(6):821-832

Scanu AM (1978) Ultrastructure of serum high density lipoproteins: facts and models. Lipids 13 (12):920-925

Schaefer EJ, Asztalos BF (2007) Increasing high-density lipoprotein cholesterol, inhibition of cholesteryl ester transfer protein, and heart disease risk reduction. Am J Cardiol 100(11A): S25-S31

Schaefer EJ et al (1979) The composition and metabolism of high density lipoprotein subfractions. Lipids 14(5):511-522

Schaefer EJ et al (2010) Marked HDL deficiency and premature coronary heart disease. Curr Opin Lipidol 21(4):289-297

Scherer M et al (2010) Sphingolipid profiling of human plasma and FPLC-separated lipoprotein fractions by hydrophilic interaction chromatography tandem mass spectrometry. Biochim Biophys Acta 1811(2):68-75

Schjoldager KT et al (2012) Probing isoform-specific functions of polypeptide GalNActransferases using zinc finger nuclease glycoengineered SimpleCells. Proc Natl Acad Sci USA 109(25):9893-9898

Schonfeld G et al (1976) Structure of high density lipoprotein. The immunologic reactivities of the COOH- and NH2-terminal regions of apolipoprotein A-I. J Biol Chem 251(13):3921-3926

Segrest JP (1977) Amphipathic helixes and plasma lipoproteins: thermodynamic and geometric considerations. Chem Phys Lipids 18(1):7-22

Segrest JP et al (1974) A molecular theory of lipid-protein interactions in the plasma lipoproteins. FEBS Lett 38(3):247-258

Segrest JP et al (1994) The amphipathic alpha helix: a multifunctional structural motif in plasma apolipoproteins. Adv Protein Chem 45:303-369

Segrest JP et al (1999) A detailed molecular belt model for apolipoprotein A-I in discoidal high density lipoprotein. J Biol Chem 274(45):31755-31758

Segrest JP et al (2000) Detailed molecular model of apolipoprotein A-I on the surface of highdensity lipoproteins and its functional implications. Trends Cardiovasc Med 10(6):246-252

Segrest JP et al (2013) MD simulations suggest important surface differences between reconstituted and circulating spherical HDL. J Lipid Res 54(10):2718-2732

Sevvana M et al (2009) Serendipitous fatty acid binding reveals the structural determinants for ligand recognition in apolipoprotein M. J Mol Biol 393(4):920-936

Shah AS et al (2013) Proteomic diversity of high density lipoproteins: our emerging understanding of its importance in lipid transport and beyond. J Lipid Res 54(10):2575-2585

Shao B (2012) Site-specific oxidation of apolipoprotein A-I impairs cholesterol export by ABCA1, a key cardioprotective function of HDL. Biochim Biophys Acta 1821(3):490-501

Shen BW et al (1977) Structure of human serum lipoproteins inferred from compositional analysis. Proc Natl Acad Sci USA 74(3):837-841

Shiflett AM et al (2005) Human high density lipoproteins are platforms for the assembly of multicomponent innate immune complexes. J Biol Chem 280(38):32578-32585 
Shimano H (2009) ApoAII controversy still in rabbit? Arterioscler Thromb Vasc Biol 29 (12):1984-1985

Silva RA et al (2005) A mass spectrometric determination of the conformation of dimeric apolipoprotein A-I in discoidal high density lipoproteins. Biochemistry 44(24):8600-8607

Silva RA et al (2008) Structure of apolipoprotein A-I in spherical high density lipoproteins of different sizes. Proc Natl Acad Sci USA 105(34):12176-12181

Sloop CH et al (1983) Characterization of dog peripheral lymph lipoproteins: the presence of a disc-shaped "nascent" high density lipoprotein. J Lipid Res 24(11):1429-1440

Sparks DL et al (1992) The conformation of apolipoprotein A-I in discoidal and spherical recombinant high density lipoprotein particles. 13C NMR studies of lysine ionization behavior. J Biol Chem 267(36):25830-25838

Stahlman M et al (2013) Dyslipidemia, but not hyperglycemia and insulin resistance, is associated with marked alterations in the HDL lipidome in type 2 diabetic subjects in the DIWA cohort: impact on small HDL particles. Biochim Biophys Acta 1831(11):1609-1617

Sung HJ et al (2012) Large-scale isotype-specific quantification of Serum amyloid A 1/2 by multiple reaction monitoring in crude sera. J Proteomics 75(7):2170-2180

Tardieu A et al (1976) Structure of human serum lipoproteins in solution. II. Small-angle x-ray scattering study of HDL and LDL. J Mol Biol 101(2):129-153

Tselepis AD et al (1995) PAF-degrading acetylhydrolase is preferentially associated with dense LDL and VHDL-1 in human plasma. Catalytic characteristics and relation to the monocytederived enzyme. Arterioscler Thromb Vasc Biol 15(10):1764-1773

Usami Y et al (2011) Detection of chymase-digested C-terminally truncated apolipoprotein A-I in normal human serum. J Immunol Methods 369(1-2):51-58

Usami Y et al (2013) Identification of sites in apolipoprotein A-I susceptible to chymase and carboxypeptidase A digestion. Biosci Rep 33(1):49-56

Utermann G (1975) Isolation and partial characterization of an arginine-rich apolipoprotein from human plasma very-low-density lipoproteins: apolipoprotein E. Hoppe Seylers Z Physiol Chem 356(7):1113-1121

Vaisar T (2009) Thematic review series: proteomics. Proteomic analysis of lipid-protein complexes. J Lipid Res 50(5):781-786

Vaisar T et al (2007) Shotgun proteomics implicates protease inhibition and complement activation in the antiinflammatory properties of HDL. J Clin Invest 117(3):746-756

Vieu C et al (1996) Identification and quantification of diacylglycerols in HDL and accessibility to lipase. J Lipid Res 37(5):1153-1161

Wald JH et al (1990) Structure of apolipoprotein A-I in three homogeneous, reconstituted high density lipoprotein particles. J Biol Chem 265(32):20037-20043

Wiesner P et al (2009) Lipid profiling of FPLC-separated lipoprotein fractions by electrospray ionization tandem mass spectrometry. J Lipid Res 50(3):574-585

Wlodawer A et al (1979) High-density lipoprotein recombinants: evidence for a bicycle tire micelle structure obtained by neutron scattering and electron microscopy. FEBS Lett 104 (2):231-235

Wolfrum C et al (2005) Apolipoprotein M is required for prebeta-HDL formation and cholesterol efflux to HDL and protects against atherosclerosis. Nat Med 11(4):418-422

Wopereis S et al (2003) Apolipoprotein C-III isofocusing in the diagnosis of genetic defects in O-glycan biosynthesis. Clin Chem 49(11):1839-1845

Wroblewska M et al (2009) Phospholipids mediated conversion of HDLs generates specific apoAII pre-beta mobility particles. J Lipid Res 50(4):667-675

$\mathrm{Wu} \mathrm{Z}$ et al (2007) The refined structure of nascent HDL reveals a key functional domain for particle maturation and dysfunction. Nat Struct Mol Biol 14(9):861-868

Wu Z et al (2009) Double superhelix model of high density lipoprotein. J Biol Chem 284(52): 36605-36619

$\mathrm{Wu} \mathrm{Z}$ et al (2011) The low resolution structure of ApoA1 in spherical high density lipoprotein revealed by small angle neutron scattering. J Biol Chem 286(14):12495-12508 
Wurfel MM et al (1994) Lipopolysaccharide (LPS)-binding protein is carried on lipoproteins and acts as a cofactor in the neutralization of LPS. J Exp Med 180(3):1025-1035

Yu B et al (1997) Lipopolysaccharide binding protein and soluble CD14 catalyze exchange of phospholipids. J Clin Invest 99(2):315-324

Zannis VI et al (1980) Isoproteins of human apolipoprotein A-I demonstrated in plasma and intestinal organ culture. J Biol Chem 255(18):8612-8617

Zerrad-Saadi A et al (2009) HDL3-mediated inactivation of LDL-associated phospholipid hydroperoxides is determined by the redox status of apolipoprotein A-I and HDL particle surface lipid rigidity: relevance to inflammation and atherogenesis. Arterioscler Thromb Vasc Biol 29(12):2169-2175

Zhang L et al (2011) Morphology and structure of lipoproteins revealed by an optimized negativestaining protocol of electron microscopy. J Lipid Res 52(1):175-184

Zhaorigetu S et al (2008) ApoL1, a BH3-only lipid-binding protein, induces autophagic cell death. Autophagy 4(8):1079-1082

Zhou W et al (2009) An initial characterization of the serum phosphoproteome. J Proteome Res 8(12):5523-5531 